\title{
Penalized partial least squares for pleiotropy
}

\author{
Camilo Broc ${ }^{1,2^{*}}\left(\mathbb{D}\right.$, Therese Truong ${ }^{3,4}$ and Benoit Liquet ${ }^{2,5}$
}

\author{
*Correspondence: \\ camilo.broc@gmail.com \\ 1 LIST, CEA, Laboratory \\ for Data Sciences \\ and Decision (Digiteo) \\ Gif-sur-Yvette, France \\ Full list of author information \\ is available at the end of the \\ article
}

\begin{abstract}
Background: The increasing number of genome-wide association studies (GWAS) has revealed several loci that are associated to multiple distinct phenotypes, suggesting the existence of pleiotropic effects. Highlighting these cross-phenotype genetic associations could help to identify and understand common biological mechanisms underlying some diseases. Common approaches test the association between genetic variants and multiple traits at the SNP level. In this paper, we propose a novel gene- and a pathway-level approach in the case where several independent GWAS on independent traits are available. The method is based on a generalization of the sparse group Partial Least Squares (sgPLS) to take into account groups of variables, and a Lasso penalization that links all independent data sets. This method, called joint-sgPLS, is able to convincingly detect signal at the variable level and at the group level.

Results: Our method has the advantage to propose a global readable model while coping with the architecture of data. It can outperform traditional methods and provides a wider insight in terms of a priori information. We compared the performance of the proposed method to other benchmark methods on simulated data and gave an example of application on real data with the aim to highlight common susceptibility variants to breast and thyroid cancers.
\end{abstract}

Conclusion: The joint-sgPLS shows interesting properties for detecting a signal. As an extension of the PLS, the method is suited for data with a large number of variables. The choice of Lasso penalization copes with architectures of groups of variables and observations sets. Furthermore, although the method has been applied to a genetic study, its formulation is adapted to any data with high number of variables and an exposed a priori architecture in other application fields.

Keywords: Genetic epidemiology, High dimensional data, Lasso Penalization, Metaanalysis, Oncology, Partial Least Square, Pathway analysis, Pleiotropy, Sparse methods, Variable selection

\section{Background}

Genome-wide association studies (GWAS) have identified numerous genetic markers linked to multiple phenotypes, suggesting the existence of pleiotropy that occurs when a single variant or gene can influence several phenotype traits [1-4]. Highlighting pleiotropy provides opportunities for understanding the shared genetic underpinnings among associated diseases. However genetic information may be spread among different studies (a) because the signal is small and larger sample sizes can increase the ability

(C) The Author(s) 2021. Open Access This article is licensed under a Creative Commons Attribution 4.0 International License, which permits use, sharing, adaptation, distribution and reproduction in any medium or format, as long as you give appropriate credit to the original author(s) and the source, provide a link to the Creative Commons licence, and indicate if changes were made. The images or other third party material in this article are included in the article's Creative Commons licence, unless indicated otherwise in a credit line to the material. If material is not included in the article's Creative Commons licence and your intended use is not permitted by statutory regulation or exceeds the permitted use, you will need to obtain permission directly from the copyright holder. To view a copy of this licence, visit http:// creativecommons.org/licenses/by/4.0/. The Creative Commons Public Domain Dedication waiver (http://creativecommons.org/publi cdomain/zero/1.0/) applies to the data made available in this article, unless otherwise stated in a credit line to the data. 
of detection (b) because in the case of rare phenotype, analyses require to study distinct data sets corresponding to different phenotypes. Therefore combining data across studies is necessary for cross-phenotype or pleiotropic association analyses. Combining data across studies on different phenotypes could also permit to increase statistical power to detect new signals weakly associated to several phenotypes. This leads to consider data sets from different sources, having common genotype data, but which phenotype traits may differ from one study to another.

In this article, we are interested in meta-analysis methods dealing with data from independent studies. Genetic information comes from single nucleotide polymorphisms (SNP). Genes are defined by a set of SNPs grouped in the same location in the genetic sequence. Pathways are groups of genes involved in a common biological mechanism. Genetic analyses aim at testing the association between genetic variants and phenotypes at the SNP-, gene- or pathway-level. Hence, information about independent data sets gives an architecture in terms of observation sets while information about either genes or pathways gives an architecture in term of groups of variables. The challenge of pleiotropy is then to take advantage of these architectures.

In addition, possible biases between observation sets can be induced in genetic studies especially due to differences of studied population, used technologies or experimented protocols. Those called "batch effects" are a common problem for meta-analyses [5], and methods for pleiotropy must take it into account. Furthermore, such methods must cope with the case where a genetic variable have a positive effect on one trait and a negative effect on another traits. Those opposite effects cannot be highlighted by standard meta-analysis methods $[6,7]$.

Various statistical methods were proposed for gene set analysis or to analyze pleiotropy. Recent pleiotropy analyses rely on statistical methods coming from gene set analysis combined with a meta-analysis [1, 8-10]. A non-exhaustive list of gene set methods can be given. Burden test and variance component tests have been developed to analyse rare variants [7, 11-13]. Alternatively, dimensionality reduction methods [14, 15] and Bayesian models have also been largely exploited $[16,17]$. We can also cite pairwise similarity based model [18], U-statistic models [19, 20], linear model family methods [21, 22] and network-based methods [23]. Furthermore, other omics fields are rising [24-27] and methods for genomics are often reused in those analyses [28].

We aim at integrating the meta-analysis perspective in cases of distinct data set to a gene set method framework. An extension of the sparse Partial Least Square (sPLS) method suited for meta-analysis for pleiotropy is proposed. It deals with observation sets and group of variables information while taking into account the possibility of opposite effects, i.e cases where a genetic variable has a positive effect on one trait and negative effect on other trait. As a sPLS family method, it can cope with the high number of variables. The method formulates at the same time a group-lasso resp. a joint-lasso penalization to represent the group of variables resp. the sets of observations.

PLS is a dimensionality reduction method developed by Wold [29] and that has been widely used for the analysis of data with large number of variables [30]. Applications have been done outside of genetic studies, for instance in chemometry [31] or for neuroimaging [32]. Unlike, its cousin method (PCA), the Principal Component Analysis (PCA) [33], the PLS deals with two blocks of data and this is used for genotype-phenotype analyses. Moreover its sparse extension using Lasso penalization has been successful at 
providing readable models [34]. Especially sparse group Partial Least Square can take into account group of variables as a priori information [35, 36]. For different group of studies an alternative Lasso penalization has been proposed by Obozinski [37] for a linear regression to deal with data made of different sets of observations. An adaptation of the Lasso penalization, the joint-sgPLS, has recently been proposed for the PLS [38], answering the specific of both groups of variables and sets of observations. In this article, we exploit the same idea to leverage pleiotropy effects, especially because the method copes with the challenge of detecting small possible opposite effects.

The method is compared to two well established statistical methods in genetic studies. The first one, ASSET [6] extends standard fixed-effects meta-analysis methods for detecting effects in opposite directions from a same genetic trait. The second one metaSKAT [7] permits to carry out gene-based meta-analysis extends SKAT and SKATo methods for meta-analyses.

The developed statistical approaches will be applied to real dataset for enriching our insights about the genetic mechanisms of thyroid and breast cancer types. We are interested into exploring gene-level and pathway-level associations for each cancer type as well as for both cancer types together.

\section{Methods}

\section{Notations}

Data are represented by $X \in \mathbb{R}^{n \times p}$ and $Y \in \mathbb{R}^{n \times q}$, two matrices, representing $n$ observations of $p$ predictors and $q$ independent variables. The Frobenius norm on matrices is denoted \|\|$_{F}$. We note $X^{T}$ the transpose matrix of $X$ and the cardinal of a set $S$ is noted $\# S$. The positive value of a real number $x$ is noted $(x)_{+}=\frac{|x|+x}{2}$ and is equal to the number if the number is positive and equal to zero otherwise. In general, observation sets can represent the fact that different sets of observations come from different sources and must be analyzed accordingly. For instance, data coming from different studies may present biases. Variables groups can represent a set of variables that are known or suspected to be part of a same signal. For instance, in genetics a gene defines an established group of SNP variables and pathways define established group of genes. Let us consider $M$ different sets of observations in the data. Noting, for $m \in \mathbb{N}, \mathbb{M}_{m}$ a subset of $\{1, \ldots, n\}$, let $\mathbb{M}=\left(\mathbb{M}_{m}\right)_{m=1 . . M}$ be a partition of $\{1, \ldots, n\}$ corresponding to the observation sets. We note $\# \mathbb{M}_{m}=n_{m}$. Row blocks are defined by this partition. Let us consider that variables are gathered in $K$ groups. Let $\mathbb{P}=\left(\mathbb{P}_{k}\right)_{k=1 . . K}$ be a partition of $\{1, \ldots, p\}$ corresponding to this variable group architecture. We note $\# \mathbb{P}_{k}=p_{k}$. We then we have $\sum_{k=1}^{K} p_{k}=p$. Column blocks are defined by this partitions. Both observation set architecture and variable group architecture can be defined at the same time as shown in Fig. 1. For matrices, the notation * is used to refer to blocks of matrices. For instance $X ., \mathbb{P}_{k}$ is the block of matrix of $X$ corresponding the columns of the $k$-th group of variables and $X_{\mathbb{M}_{m}}$, is the block of matrix of $X$ corresponding the columns of the $m$-th set of observations.

\section{Sparse Partial Least Square for structured data}

In the literature, several formulations of the PLS exist [39]. While they can have similar performances [40], PLS1 [41] has prevailed in last developments [35, 40, 41]. In the scope of this article, this formulation has been chosen in order to be able to pursue the path 


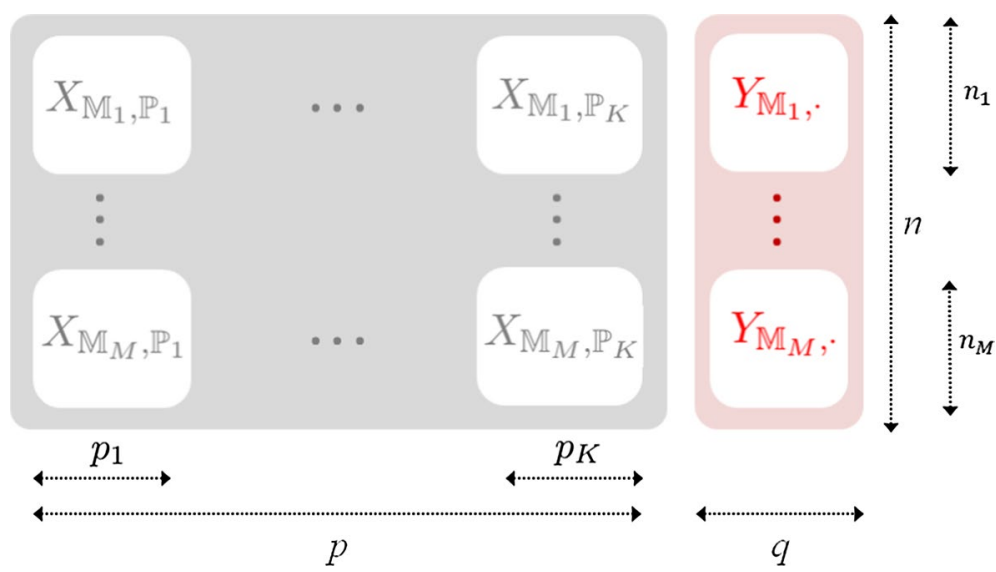

Fig. 1 Illustration of data structured by groups of variables and sets of observations. Variables and observations are assumed to be ordered by resp. groups of variables and observations sets. The notation $p$ represents the number of variables of matrix $X, q$ the number of variables of matrix $Y, n$ is the number of observations. $n_{1}, \cdots, n_{M}$ are the resp. number of observations of each observation set. $p_{1}, \cdots, p_{K}$ are the resp. number of variables in each group of variables

of previous methods. PLS finds successively couples of vector $\left\{u_{1}, v_{1}\right\}, \ldots,\left\{u_{r}, v_{r}\right\}$ for $r<\operatorname{rank}(X)$, where the couples are composed of vectors of length resp. $p$ and $q$, maximizing $\operatorname{Cov}\left(X u_{i}, Y v_{i}\right)$ for any $i \in\{1, \ldots, r\}$, under the constraint that $u_{1}, \ldots, u_{r}$ are related to orthogonal families of components [29]. It can be solved considering successive maximization problems [42], for $h \in\{1, \ldots, r\}$

$$
\max _{\left\|u_{h}\right\|\left\|_{2}=\right\| v_{h} \|_{2}=1} \operatorname{Cov}\left(X^{(h-1)} u_{h}, Y^{(h-1)} v_{h}\right)
$$

where $X_{0}=X, Y_{0}=Y$ and $X^{(h-1)}, Y^{(h-1)}$ are deflated matrices computed from $u^{(h-1)}$ $, v^{(h-1)}, X^{(h-2)}, Y^{(h-2)}$ for $h \in\{2, \ldots, r\}$. The deflation depends on the PLS mode that is chosen $[29,43]$. In the following, the notation $h$ is removed in order to simplify the formulation because we are interested in only one of the $r$ steps of the PLS.

The sparse PLS (sPLS) propose to add a penalization to the loading vectors $u$ and $v$. The following equivalence is used:

$$
\underset{\|u\|_{2}=\|v\|_{2}=1, u \in \mathbb{R}^{p}, v \in \mathbb{R}^{q}}{\operatorname{argmax}} \operatorname{Cov}(X u, Y v)=\underset{\|u\|_{2}=\|v\|_{2}=1, u \in \mathbb{R}^{p}, v \in \mathbb{R}^{q}}{\operatorname{argmin}}\left\|X^{T} Y-u v^{T}\right\|_{F}^{2}
$$

and the proof can be found in [35].

The sPLS [42] can be written as

$$
\left\{u^{(o p t)}, v^{(o p t)}\right\}=\underset{\|u\|_{2}=\|v\|_{2}=1, u \in \mathbb{R}^{p}, v \in \mathbb{R}^{q}}{\operatorname{argmin}}\left\|X^{T} Y-u v^{T}\right\|_{F}^{2}+\underbrace{\lambda P(u)}_{\begin{array}{c}
\text { Lasso Penalty term } \\
\text { for sparse PLS }
\end{array}} .
$$

The sparse PLS introduces a penalization in this formulation of the problem. The penalty $P(\cdot)$ forces smallest participation to $u$ to be set to zero. The parameter controlling the degree of sparsity in the model is $\lambda$. In the presented formula the sparsity is applied only to the vector $u$, but a similar penalization can be defined for $v$. In the context of this article we treat only the penalization of $u$ but all the results stand also for a $v$ penalization. 
Remark 1 Before analysis, the $\mathrm{X}$ and $\mathrm{Y}$ matrices are transformed by subtracting their column averages. Scaling each column by their mean and standard deviation is also often recommended [44]. Thus, the cross-product matrix $X^{T} Y$ is proportional to the empirical covariance between $\mathrm{X}$ - and $\mathrm{Y}$-variables when the columns of $\mathrm{X}$ and $\mathrm{Y}$ are centered. When the columns are standardized, $X^{T} Y$ is proportional to the empirical correlations between $\mathrm{X}$ - and $\mathrm{Y}$-variables. In this article the standardization is an important step to overcome the issue of the "batch effect" or to aggregate observations from different studies. The point has been discussed in [38].

Remark 2 Presented framework deals with the estimation of a pair of weight vectors $(u, v)$, which is the main contribution of the method in terms of methodology. This estimation step can then be included in the global framework of PLS with the deflation steps for modeling several components.

\section{Extensions of the sparse Partial Least Square}

In the following, extensions of the sPLS taking into account an observation or/and variable set architectures are presented. The last method has been recently developed [38] and deals with both kinds of architecture. It is the main topic of the article. Proposed model is an extension of the multigroup sPLS proposed by Eslami et al. [40].

In order to cope with the architectures, sgPLS has been proposed [35]:

$$
\begin{aligned}
& \left\{u^{(\text {opt })}, v^{(\text {opt })}\right\}=\underset{\|u\|_{2}=\|v\|_{2}=1, u \in \mathbb{R}^{p}, v \in \mathbb{R}^{q}}{\operatorname{argmin}}\left\|Z-u v^{T}\right\|_{F}^{2}+\lambda(1-\alpha) P_{\text {group }}(u)+ \\
& \lambda \alpha P_{\text {variable }}(u) \\
& \text { with } P_{\text {group }}(u)=\sum_{k=1}^{K} \sqrt{p_{k}}\left\|u_{\mathbb{P}_{k}}\right\|_{2}, P_{\text {variable }}(u)=\sum_{i=1}^{p}\left\|u_{i}\right\|_{2}
\end{aligned}
$$

and $Z=X^{T} Y$.

where the loading vectors $u$ and $v$ are composed of resp. $p$ and $q$ elements. Penalization $P_{\text {variable }}$ shrinks variables individually towards zero whereas penalization $P_{\text {group }}$ shrinks whole groups of variables towards zero. The parameter driving the degree of sparsity of the model is $\lambda$ whereas the parameter controlling the balance between both kinds of sparsity is $\alpha$. In this model elements of $u$ corresponding to least relevant variables and least relevant groups of variables are set to zero.

An extension using the joint Lasso penalization from Obozinski ( [37]) has been proposed [38]. This method is the object of study of this article. Its formulation for the sgPLS is: 


$$
\begin{aligned}
& \left\{U^{(\text {opt })}, V^{(\text {opt })}\right\}=\underset{\substack{U \in \mathbb{R}^{p \times M} \text { and } V \in \mathbb{R} q \times M \\
\|U, m\|_{2}=\|V, m\|_{2}=1 \text { for } m \in\{1, \cdots, M\}}}{\operatorname{argmin}} \sum_{m=1}^{M}\left\|Z^{(m)}-U U_{\cdot, m} V_{\cdot, m}\right\|_{F}^{2} \\
& \quad+\lambda(1-\alpha) P_{\text {group }}(U)+\lambda \alpha P_{\text {variable }}(U) \\
& \text { with } P_{\text {group }}(U)=\sum_{k=1}^{K} \sqrt{p_{k}}\left\|U_{\mathbb{P}_{k}, \cdot}\right\|_{F}, P_{\text {variable }}(U)=\sum_{i=1}^{p}\left\|U_{i, \cdot}\right\|_{2} \\
& \text { and } Z^{(m)}=X_{\mathbb{M}_{m},}^{T} Y_{\mathbb{M}_{m}, \cdot},
\end{aligned}
$$

where the set of loadings $U$ is composed of $p \times m$ elements ( $p$ elements per $U_{\cdot, m}$ ). The set of loadings $V$ is composed of $q \times m$ elements $(q$ elements per $V, m)$. In this model elements of $U$ corresponding to least relevant variables and least relevant group of variables are set to zero. Variables and groups of variables corresponding to least participating variables are set to zero for all $U_{., m}, m \in\{1, \ldots, M\}$ at the same time.

The solution of Eq. 5 is:

$$
\begin{aligned}
& U_{\mathbb{P}_{k}, \cdot}^{(\text {opt })}=U_{\mathbb{P}_{k}, \cdot}^{(1)}\left(1-\frac{\lambda(1-\alpha))}{2\left\|U_{\left(\mathbb{P}_{k}, \cdot\right)}^{(1)}\right\|_{F}}\right)_{+} \\
& \text {with } U_{i, \cdot}^{(1)}=U_{i, \cdot}^{(0)}\left(1-\frac{\lambda \alpha}{2\left\|U_{i, \cdot}^{(0)}\right\|_{2}}\right)_{+}
\end{aligned}
$$

and with $U_{\cdot, m}^{(0)}=X_{\mathbb{M},}^{T}, Y_{\mathbb{M},}$

where the positive value of a real number $x$ is noted $(x)_{+}=\frac{|x|+x}{2}$.

The solution is computed in 3 steps. First step (Eq. 6a) represents the solution of simple PLS for each M studies separately. Second step (Eq. 6b) applies sparsity on each variable for all studies at once. Third step (Eq. 6c) sets a sparsity on each group of variables for all studies at once. For all sparse methods, optimal parameters driving the penalization $(\lambda$ and $\alpha$ ) must be chosen. A K-fold cross-validation is used here. For each set of penalization parameters that must be tested:

- Observations are split into a partition of $L$ samples: $\left\{\mathbb{S}_{1}, \cdots, \mathbb{S}_{L}\right\}$. For a qualitative outcome, samples are chosen respecting the proportion of population of the outcome. For $l \in\{1, \cdots, L\}$, the subset of $\{1, \cdots, n\}$ where $\mathbb{S}_{l}$ is omitted is noted $\mathbb{S}_{-l}$.

- For $l \in\{1, \cdots, L\}$, a model is performed on $X_{\mathbb{S}_{-l},}$ and $Y_{\mathbb{S}_{-l}, .}$ From this model a prediction is performed on $X_{\mathbb{S}_{l}}$. which gives a prediction $\hat{Y}_{\mathbb{S}_{-l}, \text {. }}$ Prediction error is

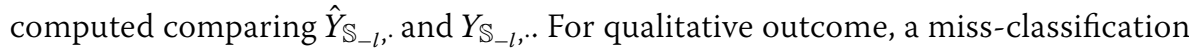
rate is computed. For a quantitative outcome a L2-norm is computed. For multivariate outcome, the mean prediction over each variable outcome is computed.

- The mean of prediction errors over the $L$ models is computed. 
The set of parameters corresponding to the lowest error of prediction over the procedure above is selected. An example of the procedure can be found in the implementation of many extensions of the sPLS [35, 36, 40].

The K-fold procedure relies on the prediction performances. However, if the signal is too small, prediction can be poor and the calculation of optimal parameters can be problematic in a cross-validation framework. Other Lasso penalization methods have struggled when the number of variables is large $[42,45]$. Due to the large number of variables in genomic data, the difference in term of prediction performance is not large enough to highlight one clear choice of penalization parameter. In this article, an alternative bootstrap strategy is proposed: sgPLS and joint-sgPLS are evaluated with given parameters on the data. Then, a bootstraps procedure is performed $B$ times. The methods sgPLS and joint-sgPLS are then implemented on each bootstrap. The selection rate for variables (resp. group of variables) over the bootstraps are calculated. Rates are considered depending on whether or not the variable is selected by the model computed on true data. Selected variables (resp. group of variable) whose rate is higher than any nonselected variables are kept in the final selection.

Remark 3 The proposed joint penalization is biconvex but not convex, and thus multiple local minima may exist. The method can then be sensible to the starting point of its algorithm. Some development using several starting points can enhance the probability of reaching a global optimum and some can even ensure it. In dimensionality reduction methods a semidefinite relaxation has been proposed which ensures the convergence [46] at the cost of computational efficiency. Methods relying on random initialization have increased the chances of finding the global optimum but with lower theoretical guarantees. Inheriting such developments for the joint-sgPLS would be interesting for future developments.

Remark 4 Group sparse dimensionality reduction methods such as sgPLS and jointsgPLS need to be extended in case of overlapping groups of variables [47]. In the scope of this article, groups of variables are supposed to be disjointed.

\section{Benchmark methods}

Both ASSET and metaSKAT are considered as benchmark methods.

ASSET is a method suited for meta-analysis providing a p-value across studies [6]. The input of the method are single variables summary statistics which are combined by the method. ASSET exhaustively explores subsets of studies for the presence of true association signals that are in either the same direction or possibly opposite directions.

For a given variable $i \in\{1, \cdots, p\}$ and a given set of studies $m \in\{1, \cdots, M\}$ the esti-

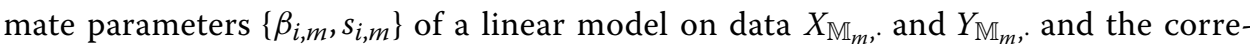
sponding statistic $Z_{i, m}=\frac{\beta_{i, m}}{s_{i, m}}$ are computed. Then for each possible subset $S \subset\{1, \cdots, M\}$, the mean statistic $Z_{i}(S)=\sum_{l \in S} \sqrt{\pi_{l}(S)} Z_{i, l}$ is evaluated with $\pi_{l}(S)=\frac{n_{l}}{\sum_{l \in S} n_{l}}$. ASSET seeks for the optimal subset of observations following the criteria $\max _{S \in \mathbb{S}}|Z(S)|$. A p-value is computed from this final statistic. ASSET relies on statistics at variable level and hence do not propose gene- or pathway-level information. 
Further, the current version of ASSET provides pleiotropy result for each variant which should be corrected using a FDR correction in order to control possible false positive pleiotropy effect.

SKAT is a method to detect association between rare variants in a region and a phenotype (continuous or binary). It is a supervised test for joint effects of multiple variants in a region on a phenotype. The metaSKAT method can do the same but aggregating several studies. This method outputs a p-value corresponding to a set of variables, for instance a gene or a pathway. The method is based on a weighted sum of SKAT statistics of the different studies [7].

The statistics $S_{m, k}=X_{\mathbb{M}_{m}, \mathbb{P}_{k}}^{T} \tilde{Y}_{\mathbb{M}_{m}}$. is computed where $\tilde{Y}$ of a generalized linear model performed on $\mathrm{Y}$ with respect to covariates. Then a weighted sum is computed on these statistics summing among the studies and then following the variables: $Q=\sum_{m=1}^{M} \sum_{k=1}^{K}\left(w_{m, k} S_{m, k}\right)^{2}$ where $w_{.,}$, are weights that must be chosen. Next, a p-value is computed. The method relies on the square of the statistic and then can detect opposite effects from one study to another.

Unlike metaSKAT, sgPLS and joint-sgPLS ASSET gives one result per variables, and does not give information for a whole group of variables. We can note that both ASSET and metaSKAT are p-value oriented method which allow them to select variables. However, they cannot propose predictions whereas joint-sgPLS can.

\section{Simulated data}

Presented methods are illustrated on simulated data presenting the architecture given in Fig. 1. From one side, SNP genotypes are coded as minor allele counting $\{0,1,2\}$ and a certain correlation is expected within a group of SNP from the same linkage disequilibrium block. From the other side, phenotype data are binary and have a true effect from one or more genetic markers. In order to simulate the correlation between SNPs, for a group of variables $\mathbb{P}_{k}$, a multivariate normal distribution with $n$ observations $\boldsymbol{x}_{k}^{\text {(continuous) }} \sim \mathcal{N}_{p_{k}}\left(\mu_{k}, \Sigma_{k}\right)$ is simulated where $\mu_{k}$ is a null vector of size $p_{k}$ and $\Sigma_{k}$ is a $p_{k} \times p_{k}$ matrix with 1 on the diagonal and $\rho_{k}$, coefficients controlling the correlation between SNPs within a group, outside of the diagonal. A simulation of this variable gives a matrix which represents simulated observations for group of variables $k$. Those blocks are concatenated in a $n \times p$ matrix, $X^{(\text {continuous })}$ that represents the whole data.

In order to have $\{0,1,2\}$ genotype data, a discretization is performed. For a given variable $j \in \mathbb{P}_{k}$, we aim at simulating a SNP variable with a Minor Allele Frequency (MAF), which we note $\mathrm{MAF}_{j}$. This MAF means that:

$$
\begin{aligned}
& P\left(x_{j}=0\right)=\left(1-\mathrm{MAF}_{j}\right)^{2} \\
& P\left(x_{j}=1\right)=2 \operatorname{MAF}_{j}\left(1-\mathrm{MAF}_{j}\right) \\
& P\left(x_{j}=2\right)=\operatorname{MAF}_{j}^{2} .
\end{aligned}
$$

To this aim, for a given $\mathrm{MAF}_{j}$, quantiles $q_{1}^{(j)}$ and $q_{2}^{(j)}$ are chosen such as $P\left(x_{j} \leq q_{1}\right)=\left(1-M A F_{j}\right)^{2}$ and $P\left(x_{j} \leq q_{2}\right)=\left(1-M A F_{j}\right)^{2}+2 M A F_{j}\left(1-M A F_{j}\right)$

A discrete genotype, $X^{(\text {discrete })}$, is computed such that 


$$
X_{i, j}^{(\text {discrete })}=\left\{\begin{array}{l}
0 \text { if } X_{i, j}^{(\text {continuous })} \leq q_{1}^{(j)} \\
1 \text { if } q_{1}^{(j)} \leq X_{i, j}^{(\text {continuous })} \leq q_{2}^{(j)} \\
2 \text { if } X_{i, j}^{(\text {continuous })}>q_{2}^{(j)},
\end{array}\right.
$$

where $i \in\{1, \cdots, n\}$ are simulated observations and $j \in \mathbb{P}_{k}$ is a variable of $k$-th group of variables.

For each observation $i$, a binary phenotype $y_{i}$ is simulated with a logit model

$$
\operatorname{logit}\left(\pi_{i}\right)=\log \left(\frac{\pi_{i}}{1-\pi_{i}}\right)=\alpha+\sum_{j=1}^{p} X_{i, j}^{(\text {discrete })} \beta_{j},
$$

where $\pi_{i}=P\left(y_{i}=1 \mid\right.$ data $), \beta_{j}$ for $j \in\{1, \cdots, p\}$ is a regression parameter.

Then different simulations of the process can be performed successively in order to simulate several studies.

\section{Results}

The code used for running the methods is available on github (https://github.com/camil obroc/BMC_joint_sgPLS).

\section{Simulation}

In this article, simulated genotype has 25 groups of 20 variables. There are then 500 variables and data is composed of two studies with equal number of observations. Combinations of parameters are considered to study a variation of (i) the existence of opposite effects from one observation set to another (ii) the portion of SNPs of the groups having effects (iii) the sample size. Values choice are given in Table 1. Variation (i) permits to see the ability of method at detecting a signal even when opposite effects occur. Variation (ii) allows to observe the influence of intra-group sparsity on the performances of the methods. Variation (iii) shows cases where the signal is easier or harder to retrieve due to the different sample sizes.

The intra-group correlation parameters $\rho_{k}$ are equal to 0.5 and the MAF is equal to 0.3 for each variable. The first 5 groups have an effect in the model of the simulations. For each group, half of the non-null regression parameters are positives (taken at random) while the other half is negative. In cases where all SNPs have effects (cases 1, 2, 5 and 6),

Table 1 Values used for the 8 cases of simulated data

\begin{tabular}{llll}
\hline Case & $\begin{array}{l}\text { Opposite direction } \\
\text { effect }\end{array}$ & $\begin{array}{l}\text { Percent of SNPs having an effect } \\
\text { in groups having an effect }\end{array}$ & $\begin{array}{l}\text { Total number } \\
\text { of observations }\end{array}$ \\
\hline 1 & No & $100 \%$ & 200 \\
2 & No & $100 \%$ & 400 \\
3 & No & $50 \%$ & 200 \\
4 & No & $50 \%$ & 400 \\
5 & Yes & $100 \%$ & 200 \\
6 & Yes & $100 \%$ & 400 \\
7 & Yes & $50 \%$ & 200 \\
\hline
\end{tabular}


the absolute value of those parameters is set to exp (0.1) whereas in cases with half of SNPs having effects (cases 3, 4, 7 and 8), the absolute value of those parameters is set to $\exp (0.5)$.

For all methods 50 replications of the data are performed. For the implementation of the sgPLS and joint-sgPLS, penalisation parameters must be chosen similarly to [35]. The penalization parameter $\lambda$ and $\alpha$ are optimized through a $K$-fold penalization procedure with an error of prediction as criteria. Choosing a parameter $\lambda$ is equivalent to set a number of selected groups [35]. In this simulation the grid of number of selected groups $\{1, \cdots, 25\}$ is used and the grid for $\alpha$ is $\{0.1,0.5,0.9\}$. Figures 2 and 3 show the error of prediction performances through a cross-validation procedure of the sgPLS and jointsgPLS in a simulation of case 1, for different levels for $\alpha$ and different levels of group selection. The observed mean and the variance of the error rate over 50 replications are presented. In the framework of the method the set of parameters corresponding to the lowest error of prediction rate is kept for the model.

For ASSET, sgPLS and joint-sgPLS, the variables selected by the models are compared to the variable having an effect on the true model. For metaSKAT, sgPLS and jointsgPLS, the group of variables selected by the models are compared to the group of variables having an effect on the true model.

Results of the simulations are presented in Table 1 for sgPLS, joint-sgPLS, ASSET and metaSKAT. The measures of performance are the True Positives (TP), False positives (FP), False Negatives (FN) and True Negatives (TN) (Table 2).

Considering cases 1 and 2, we can see that ASSET and metaSKAT have FP lower than TP in opposition to sgPLS and joint-sgPLS. They are then more conservative than

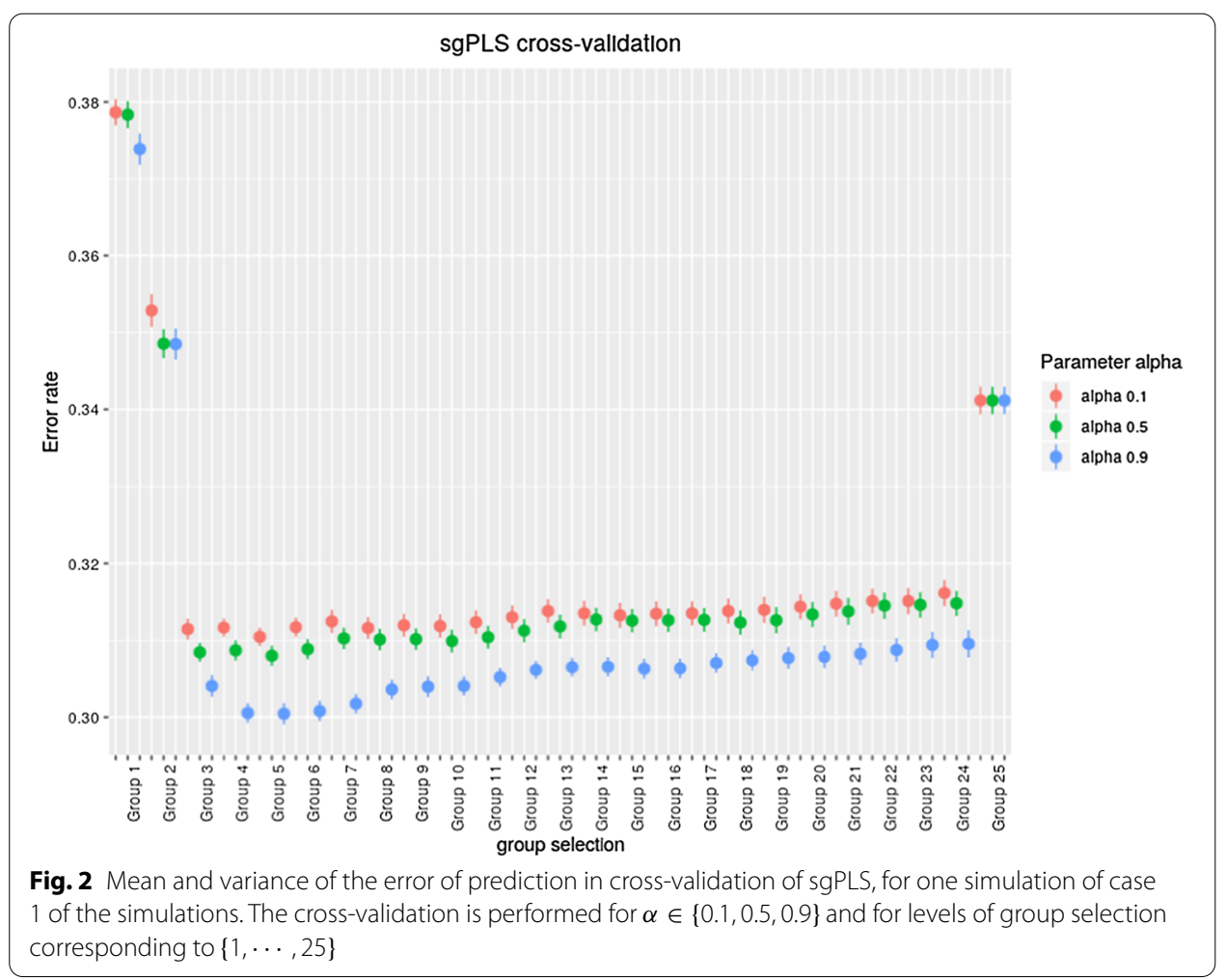




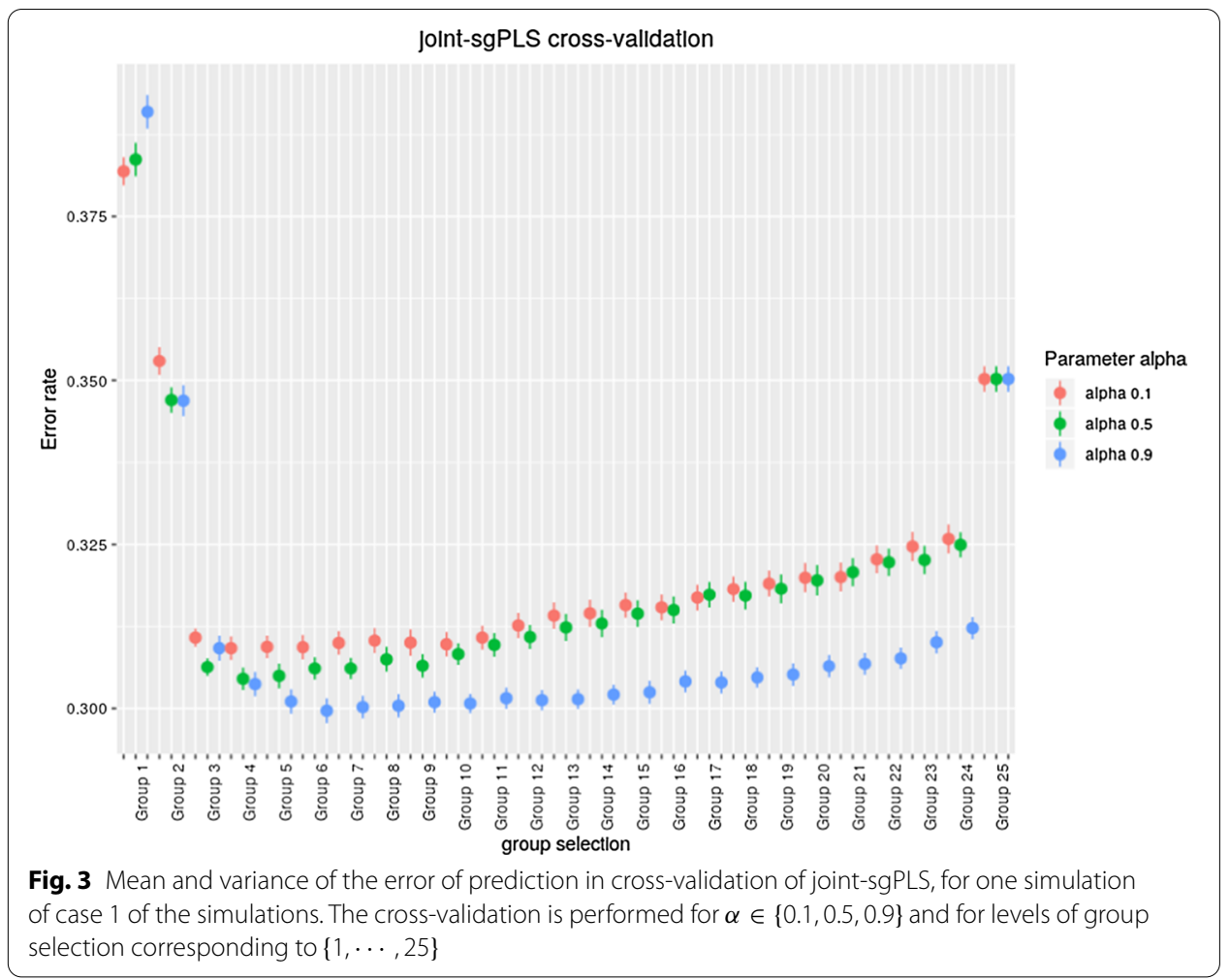

the two later methods. We can see that overall, each model performs better when the number of observations is higher (200 against 400). We can see that when the intrasparisity is set to $50 \%$ (cases 3, 4, 7 and 8) rather than $100 \%$ (cases 1, 2, 5 and 6), variable-level results for ASSET sgPLS and joint-sgPLS are inflated by more than a half. This may be due to the fact that the methods struggle to differentiate the effect of variables within a same group. The gene-level results are similar whichever the intrasparsity is for metaSKAT, sgPLS and joint-sgPLS. Cases 1, 2, 3 and 4 have effect in the same direction among studies while cases 5,6,7 and 8 show effects in opposite directions. We can see that when effects are in the same direction or opposite direction, sgPLS can compete with joint-sgPLS and with other benchmark methods while being the least conservative. On the other hand, when effects are in different directions, sgPLS performances fumble whereas other methods keep a similar TP/FP ratio. Comparing closely ASSET to jointsgPLS, we can see that joint-sgPLS have always a higher TP and the largest difference can be seen when all variables are involved within a group (cases 1, 2, 5, 6). This is probably due to the fact that joint-sgPLS can draw information at the group-level to infer single variable results. Comparing closely metaSKAT to joint-sgPLS, we can see that both methods can retrieve a large amount of groups participating to the effect. The method joint-sgPLS have always a higher TP in each cases. In cases 1 and 5, metaSKAT TP is especially low. Those are cases with the smallest number of observations and with small regression parameters $\beta_{j}$ and hence where the intensity of the signal is the lowest.

Overall, we can see that sgPLS and joint-sgPLS have competitive performances for detecting effect in the same direction while joint-sgPLS is the method with the best performance for detecting opposite effects. Furthermore sgPLS and presented joint-sgPLS 
Table 2 Performances in terms of mean number of TP, FP, FN and TN over simulation cases 1 to 8 for methods sgPLS, joint-sgPLS, ASSET and metaSKAT

\begin{tabular}{|c|c|c|c|c|c|c|c|c|c|}
\hline \multicolumn{5}{|c|}{ Variable level performances } & \multicolumn{5}{|c|}{ Group level performances } \\
\hline & TP & FP & FN & TN & & TP & FP & FN & TN \\
\hline \multicolumn{10}{|c|}{ Simulation case 1} \\
\hline sgPLS & 46.78 & 29.24 & 53.22 & 370.76 & sgPLS & 3.54 & 3.82 & 1.46 & 16.18 \\
\hline joint-sgPLS & 40.84 & 27.16 & 59.16 & 372.84 & joint-sgPLS & 3.44 & 3.78 & 1.56 & 16.22 \\
\hline ASSET & 29.98 & 22.14 & 70.02 & 377.86 & metaSKAT & 2.22 & 1.08 & 2.78 & 18.92 \\
\hline \multicolumn{10}{|c|}{ Simulation case 2} \\
\hline sgPLS & 75.76 & 139.34 & 24.24 & 260.66 & sgPLS & 4.74 & 11.9 & 0.26 & 8.1 \\
\hline joint-sgPLS & 66.12 & 76.44 & 33.88 & 323.56 & joint-sgPLS & 4.48 & 7.62 & 0.52 & 12.38 \\
\hline ASSET & 47.74 & 25.4 & 52.26 & 374.6 & metaSKAT & 3.14 & 1.26 & 1.86 & 18.74 \\
\hline \multicolumn{10}{|c|}{ Simulation case 3} \\
\hline sgPLS & 36.58 & 63.56 & 13.42 & 386.44 & sgPLS & 4.78 & 4.02 & 0.22 & 15.98 \\
\hline joint-sgPLS & 31.3 & 48.04 & 18.7 & 401.96 & joint-sgPLS & 4.5 & 3.06 & 0.5 & 16.94 \\
\hline ASSET & 29.46 & 46.88 & 20.54 & 403.12 & metaSKAT & 3.62 & 0.96 & 1.38 & 19.04 \\
\hline \multicolumn{10}{|c|}{ Simulation case 4} \\
\hline sgPLS & 42.68 & 148.78 & 7.32 & 301.22 & sgPLS & 4.88 & 11.1 & 0.12 & 8.9 \\
\hline joint-sgPLS & 40.42 & 115.96 & 9.58 & 334.04 & joint-sgPLS & 4.92 & 8.54 & 0.08 & 11.46 \\
\hline ASSET & 35.26 & 54.68 & 14.74 & 395.32 & metaSKAT & 4.18 & 1.02 & 0.82 & 18.98 \\
\hline \multicolumn{10}{|c|}{ Simulation case 5} \\
\hline sgPLS & 17.96 & 36.28 & 82.04 & 363.72 & sgPLS & 1.46 & 4.32 & 3.54 & 15.68 \\
\hline joint-sgPLS & 43.44 & 25.2 & 56.56 & 374.8 & joint-sgPLS & 3.46 & 3.5 & 1.54 & 16.5 \\
\hline ASSET & 30.58 & 22.48 & 69.42 & 377.52 & metaSKAT & 2.2 & 0.98 & 2.8 & 19.02 \\
\hline \multicolumn{10}{|l|}{ Simulation 6} \\
\hline sgPLS & 75.76 & 139.34 & 24.24 & 260.66 & sgPLS & 4.74 & 11.9 & 0.26 & 8.1 \\
\hline joint-sgPLS & 66.12 & 76.44 & 33.88 & 323.56 & joint-sgPLS & 4.48 & 7.62 & 0.52 & 12.38 \\
\hline ASSET & 47.74 & 25.4 & 52.26 & 374.6 & metaSKAT & 3.14 & 1.26 & 1.86 & 18.74 \\
\hline \multicolumn{10}{|c|}{ Simulation case 7} \\
\hline sgPLS & 13.2 & 74.96 & 36.8 & 375.04 & sgPLS & 2.04 & 6.5 & 2.96 & 13.5 \\
\hline joint-sgPLS & 35.62 & 94.24 & 14.38 & 355.76 & joint-sgPLS & 4.58 & 7.02 & 0.42 & 12.98 \\
\hline ASSET & 29.18 & 45.5 & 20.82 & 404.5 & metaSKAT & 3.54 & 0.92 & 1.46 & 19.08 \\
\hline \multicolumn{10}{|c|}{ Simulation case 8} \\
\hline sgPLS & 14.3 & 72.28 & 35.7 & 377.72 & sgPLS & 1.88 & 5.76 & 3.12 & 14.24 \\
\hline joint-sgPLS & 39.12 & 99.06 & 10.88 & 350.94 & joint-sgPLS & 4.9 & 7.18 & 0.1 & 12.82 \\
\hline ASSET & 34.04 & 56.14 & 15.96 & 393.86 & metaSKAT & 4.22 & 0.86 & 0.78 & 19.14 \\
\hline
\end{tabular}

have the merit of giving single variable results and group results in the same model. This allow variable-level results to be enhanced by the group a priori information.

\section{Pleiotropy investigation on breast and thyroid cancer}

The developed statistical approaches were applied to real data in order to enrich our insights about the genetic mechanisms involved in carcinogenesis of thyroid and breast cancers. Thyroid and breast cancers share some similarities in their biology: both are more frequent in women, are influenced by hormonal and reproductive factors and are hormonally-mediated. Moreover, individuals diagnosed with breast cancer are more likely to develop thyroid cancer as a secondary malignancy than patient diagnosed with other cancer types, and vice-versa [48]. Genetic factor contributing to the incidence of 
breast cancer have been extensively studied, and it is known that genetic variants explain approximately 49 percent of the familial risk to develop this disease. Using GWAS, 313 risk variants were identified for breast cancer [49]. On the other hand, GWAS studies on thyroid cancer have been scarce, due to the lesser incidence of this disease as well as the lack of data. However, it has been shown that thyroid cancer is the only cancer for which genetic factors contribute more than environmental factors [50]. Only 4 loci have been associated with thyroid cancer risk and have been replicated in other studies [51]. One of them, 2q35, was also previously reported to increase risk of breast cancer [52]. To date, no study has been conducted to identify common genetic factors between breast and thyroid cancer. Exploring the genetic relationship between the two cancers would help to elucidate the common mechanisms between both disease and could permit to improve their diagnostic and therapeutic management.

We propose to illustrate the methods on real datasets, by investigating the pleiotropic effect of genetic variants from candidate pathways in breast and thyroid cancers.

Beluhca dataset includes data from CECILE, a french case-control study on breast cancer (1 125 cases, 1172 controls) and from CATHY a french case-control study on thyroid cancer (463 female cases and 482 female controls). All these individuals were genotyped using a customized microarray including 8716 genetic variants from 28 candidate pathways (648 genes) selected from KEGG database and from a literature review (SNPs are located at $+/-50 \mathrm{~kb}$ from the gene boundaries). After quality controls, we retained 6677 SNPs available for both type of cancers. Missing values were imputed using the median among cases or controls and data were centered to $\mu=0$. When 2 SNPs were correlated at $r^{2}=1$, one of the SNP was removed and couple of extremely correlated $\left(r^{2}>0.98\right)$ SNPs belonging to same genes were eliminated.

As group sparse dimensionality reduction methods such as sgPLS and joint-sgPLS need to be extended in case of overlapping groups of variables [47], 10 non-overlapping pathways were selected and only the 3766 SNPs related to those groups were kept in the final database. After all these preprocessing, the new dataset is composed of 3766 SNPs, grouped in 337 non-overlapping genes and 10 non-overlapping pathways. The list of the pathways and genes is displayed in Tables 5 and 6 in Appendix 1.

The methods implemented in this article are: ASSET, metaSKAT, sgPLS and jointsgPLS. For metaSKAT, sgPLS and joint-PLS, SNP-level, gene-level and pathway-level results are given by the methods whereas ASSET gives only SNP-level results. Hence, in the case of ASSET, genes corresponding to selected SNPs are considered. For each SNP $i$, an univariate logistic model for gene-disease association can be considered separately for thyroid data and breast data (thyroid and breast cancer, Fig. 4).

As it has been presented before, for sgPLS and joint-sgPLS, a calibration of the parameters is generally performed through a cross-validation procedure. This process relies on the definition of a measure of performance: the error of prediction of the model. However, in genetic studies, the effects are small and the prediction performances based on genetic units are usually very low. The prediction performance of sgPLS and joint-sgPLS are not different enough from one set of penalization parameters to another. In order to facilitate the interpretation, we present the results for calibration parameters set to 20 genes and 3 pathways and $\alpha=0.5$. We explore the stability of the methods using the bootstrap strategy described in the section method. 


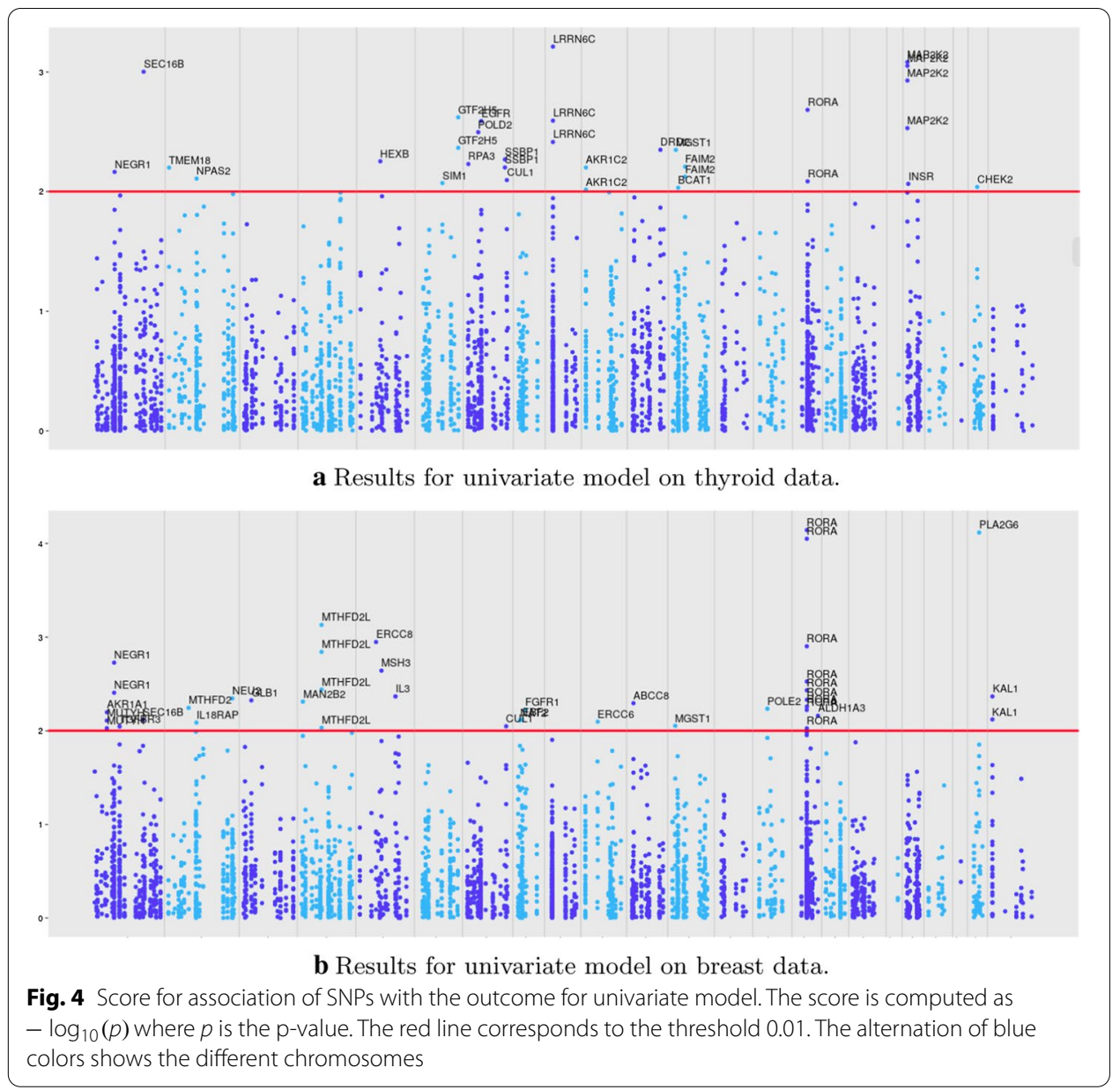

Figures 5 and 6 present this rate for preselected and non-preselected features. A gene and resp. a pathway is kept in the final selection if and only if it is preselected and its rate of selection among the bootstraps is higher than any other gene (resp. pathway) that is not preselected. We can see that for joint-sgPLS less genes are selected than for other methods (4 against resp. 20 and 18 for metaSKAT and sgPLS on both data).

Results of the selection are presented in Table 3 where the name of genes and pathways is presented. "sgPLS single" stand for the use of the sgPLS on thyroid and breast data separately while "sgPLS both" stands for the use of the method on a concatenation of both data set standardizing by study. Only genes that are selected by at least one method are presented. No genes from metabolism of xenobiotics pathway have been selected through all methods. We can see that methods focusing on SNP-level information select gene from one of the study but never both studies at the same time except for INSR which is selected for both studies for SKAT. This genes is not selected by meta-analysis methods. Genes selected by group-level methods (ASSET, metaSKAT, sgPLS, joint-sgPLS)) that are not selected by variable-level methods are: PTEN, RORA, MSH3, IL18RAP,GNPDA2, LRRN6C, NEGR1, NR3C1, SEC16B, HEXA, HEXB, MAN2B2, NEU2, TGBR3, NMNAT2, CYP2C18, CYP2C19, MGST1. Those genes are good candidates for further investigations as they are not selected by 


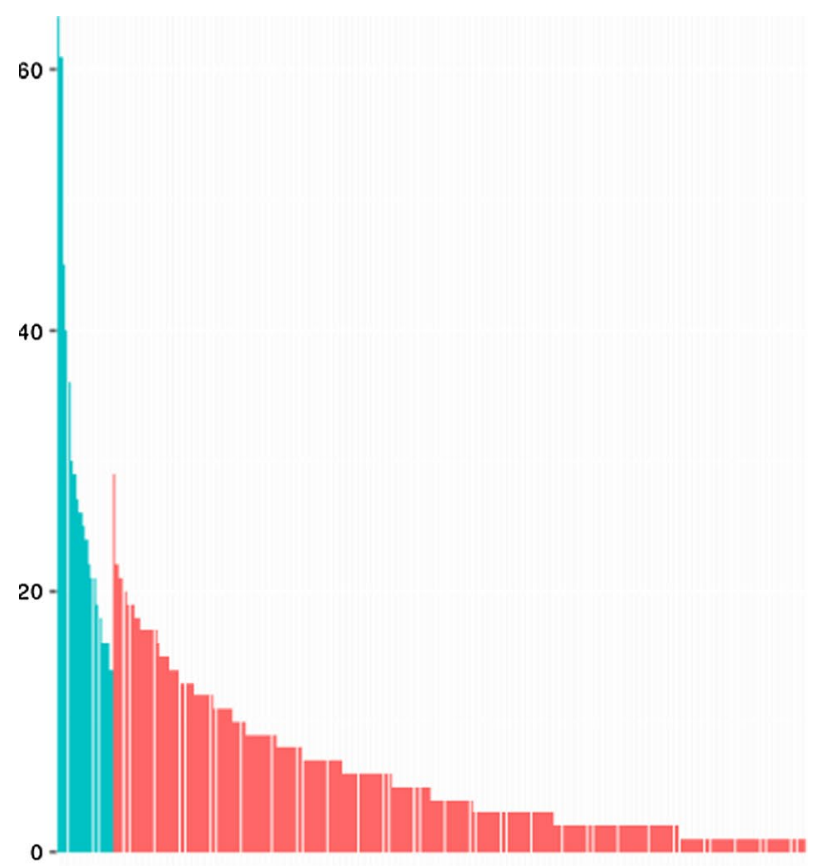

a Percent of selection of genes for sgPLS on thyroid data, 100 bootstraps

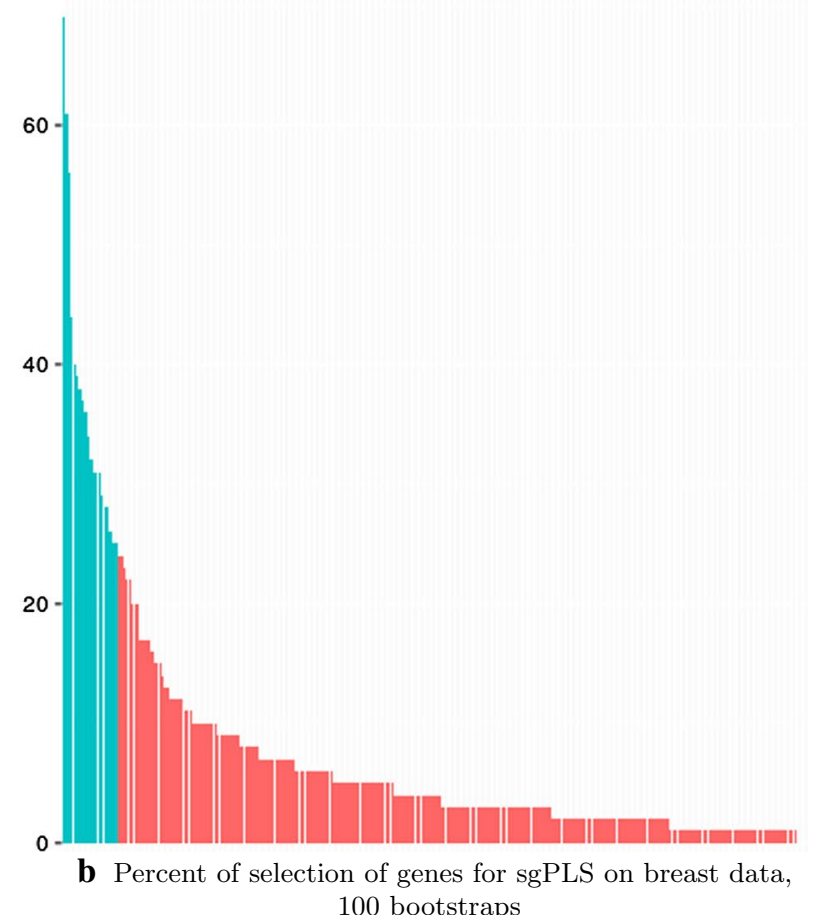

Fig. 5 Percent of selection of genes for sgPLS and joints sgPLS on 100 bootstraps. a sgPLS on thyroid data. $\mathbf{b}$ sgPLS on breast data. c sgPLS on both data. $\mathbf{d}$ joint-sgPLS. Genes selected on original data (preselected ones) are in blue while other genes (non-preselected ones) are in red

study by study analyses but are selected by meta analyses. We can note that 5 out the 8 genes selected for Obesity and obesity-related phenotypes pathway and all genes selected for Other glycan degradation are part of those genes. Those pathways would 

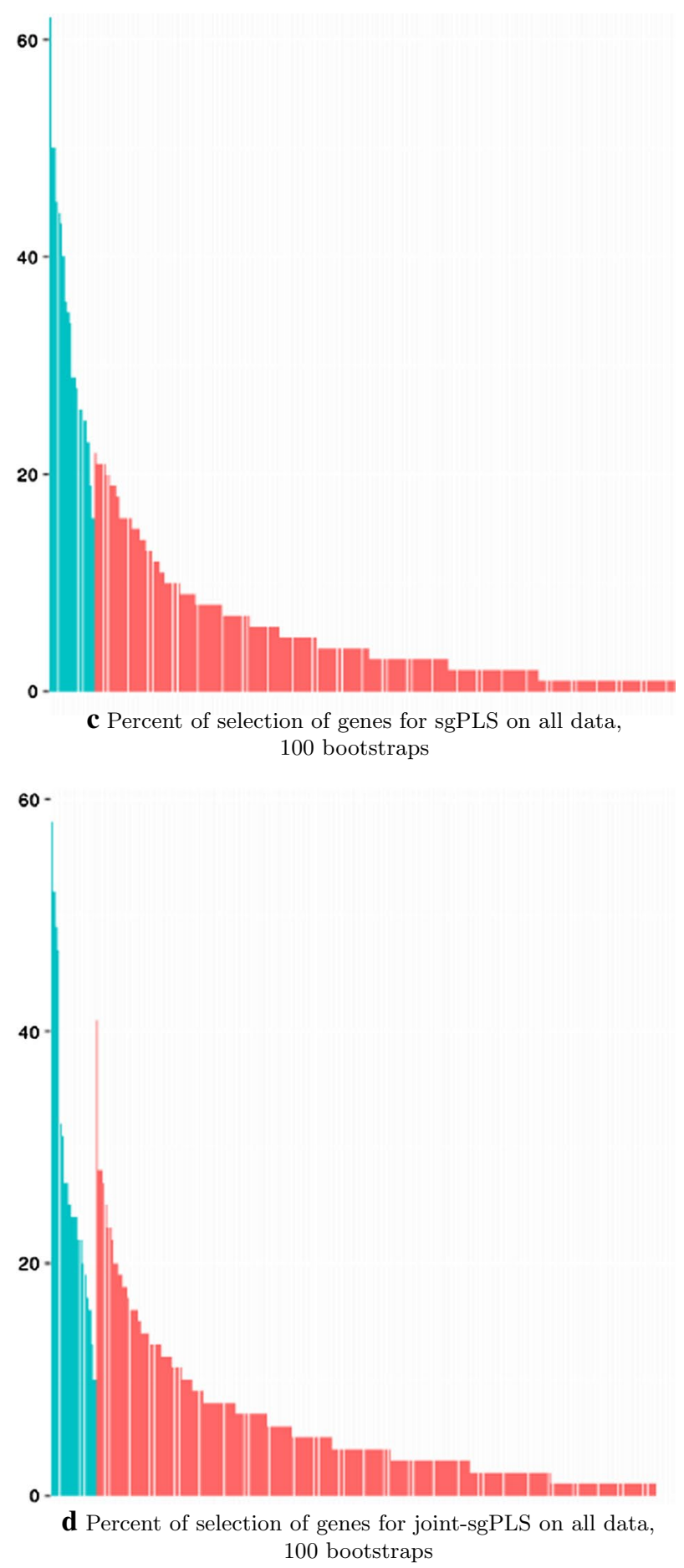

Fig. 5 continued

not have been as much hightlighted without meta-analyses. Genes selected for thyroid data sets and selected by meta-analyses are: MAP2K2, GTF2H1 and CYP2F1. Those genes are then related to thyroid cancer but meta-analyses suggest they may be involved with breast cancer in a common effect. Genes selected for breast data and 
Table 3 Selected data sets in terms of genes and pathways. Selection of resp. thyroid data set, breast data sets and both data set is represented in resp. blue, green and read

\begin{tabular}{|c|c|c|c|c|c|c|}
\hline \multirow[b]{2}{*}{ gene } & \multicolumn{2}{|c|}{ Single study analysis } & \multicolumn{2}{|c|}{ meta-analysis } & \multirow[b]{2}{*}{ sgPLS Both } & \multirow[b]{2}{*}{ joint-sgPLS } \\
\hline & SKAT & sgPLS single & ASSET & metaSKAT & & \\
\hline \multicolumn{7}{|l|}{ Cell cycle } \\
\hline Whole pathway & & thyroid & & & both & \\
\hline EGFR & & & thyroid & & & \\
\hline MAP2K2 & thyroid & thyroid & both & & & both \\
\hline PLA2G6 & breast & breast & both & & both & both \\
\hline PTEN & & & both & & & \\
\hline \multicolumn{7}{|l|}{ Circadian Rythm } \\
\hline Whole pathway & & breast & & & & \\
\hline RORA & & & both & & & \\
\hline \multicolumn{7}{|l|}{ DNA repair } \\
\hline Whole pathway & thyroid & & & & & \\
\hline ERCC3 & breast & breast & both & both & both & both \\
\hline ERCC6 & breast & breast & & both & & \\
\hline ERCC8 & breast & breast & both & & & \\
\hline FEN1 & & & & & both & \\
\hline GTF2H1 & thyroid & & & & both & \\
\hline GTF2H5 & thyroid & thyroid & thyroid & & & \\
\hline MAPK8 & & breast & & & & \\
\hline MSH3 & & & both & & & \\
\hline MUTYH & breast & breast & & both & both & \\
\hline OGG1 & breast & breast & & & & \\
\hline POLD2 & & & thyroid & & & \\
\hline POLE2 & breast & breast & & & both & \\
\hline RPA2 & & breast & & & both & \\
\hline SSBP1 & thyroid & thyroid & & & & \\
\hline \multicolumn{7}{|c|}{ Folate metabolism } \\
\hline Whole pathway & & breast & & & both & both \\
\hline MTHFD2 & breast & breast & & & both & both \\
\hline MTHFD2L & breast & breast & both & & both & \\
\hline \multicolumn{7}{|c|}{ Inflammatory response } \\
\hline Whole pathway & & breast & & & & both \\
\hline CYP4F11 & & & & both & & \\
\hline IL13 & breast & breast & & both & both & \\
\hline IL15 & thyroid & & & & & \\
\hline IL18RAP & & & both & & & \\
\hline IL1A & & breast & & & & \\
\hline IL3 & breast & breast & & & & \\
\hline MMP25 & & breast & & & both & \\
\hline \multicolumn{7}{|c|}{ Obesity and obesity-related phenotypes } \\
\hline $\begin{array}{l}\text { Whole pathway } \\
\text { DRD2 }\end{array}$ & thyroid & & & & & \\
\hline
\end{tabular}

selected by meta-analyses are: PLAG2G6, ERCC3, ERCC6, MUTYH, MTHFD2, IL13, NAT2. Meta-analyses suggest that these genes may also be involved with thyroid cancer in a common effect. We can see that joint-sgPLS selects a lower number of genes (resp.4) compared to ASSET, metaSKAT and sgPLS (resp. 19, 20, 18). Method sgPLS and joint-sgPLS select the glycan pathway and folate metabolism pathway and sgPLS 
Table 3 (continued)

\begin{tabular}{|c|c|c|c|c|c|c|}
\hline \multirow[b]{2}{*}{ gene } & \multicolumn{2}{|c|}{ Single study analysis } & \multicolumn{2}{|c|}{ meta-analysis } & \multirow[b]{2}{*}{ sgPLS Both } & \multirow[b]{2}{*}{ joint-sgPLS } \\
\hline & SKAT & sgPLS single & ASSET & metaSKAT & & \\
\hline FAIM2 & thyroid & & & & & \\
\hline GNPDA2 & & & & both & & \\
\hline INSR & both & & both & both & both & \\
\hline LRRN6C & & & both & & & \\
\hline NEGR1 & & & both & & & \\
\hline NR3C1 & & & & both & & \\
\hline SEC16B & & & both & & & \\
\hline \multicolumn{7}{|c|}{ Other glycan degradation } \\
\hline Whole pathway & & & & & both & both \\
\hline HEXA & & & & both & & \\
\hline HEXB & & & & both & & \\
\hline MAN2B2 & & & both & both & both & \\
\hline NEU2 & & & both & both & & \\
\hline \multicolumn{7}{|c|}{ Precocious or delayed puberty } \\
\hline Whole pathway & & & & both & & \\
\hline FGFR1 & breast & breast & & & & \\
\hline KAL1 & breast & & & both & & \\
\hline TGFBR3 & & & both & both & & \\
\hline \multicolumn{7}{|c|}{ Nicotinate and nicotinamide metabolism } \\
\hline Whole pathway & & & & & & \\
\hline ENPP3 & & & & both & & \\
\hline NADK & & breast & & & both & \\
\hline NMNAT2 & & & & both & & \\
\hline NMNAT3 & & & & both & & \\
\hline NT5C & thyroid & thyroid & & & & \\
\hline PNP & & & & & both & \\
\hline $\mathrm{ADH} 1 \mathrm{~A}$ & thyroid & & & & & \\
\hline AKR1A1 & breast & breast & & both & & \\
\hline AKR1C2 & thyroid & & & & & \\
\hline ALDH1A3 & breast & breast & & & both & \\
\hline CYP2C18 & & & & both & & \\
\hline CYP2C19 & & & & both & & \\
\hline CYP2E1 & thyroid & & & & & \\
\hline CYP2F1 & thyroid & thyroid & both & & & \\
\hline GSTA2 & & thyroid & & & & \\
\hline MGST1 & & & both & & both & \\
\hline NAT2 & breast & breast & both & & both & \\
\hline
\end{tabular}

selects also cell cycle pathway. PLS methods suggest that pathway-level effect could be involved.

Remark 5 Results based on different choice of calibration parameters for sgPLS and joint-sgPLS (50, 100 genes and 5 pathways) showed similar patterns. 


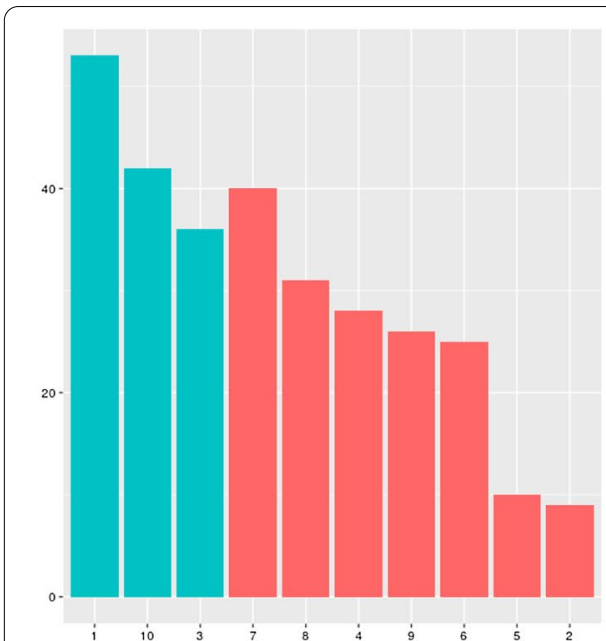

a Percent of selection of pathways for sgPLS on thyroid data, 100 bootstraps

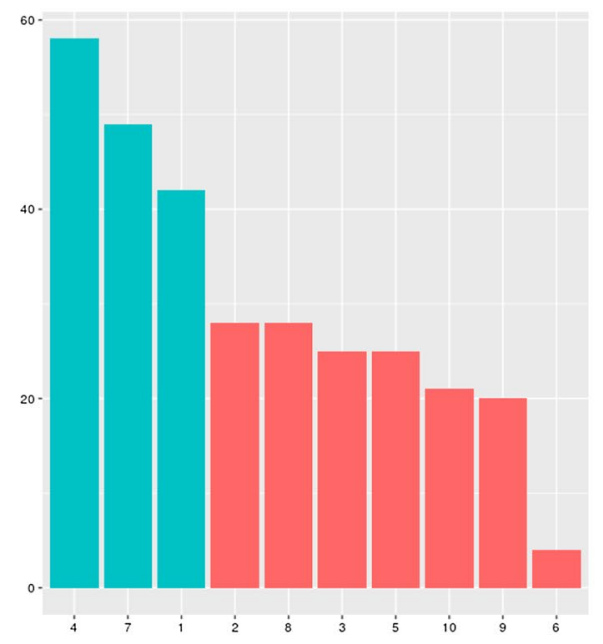

c Percent of selection of pathways for sgPLS on all data, 100 bootstraps

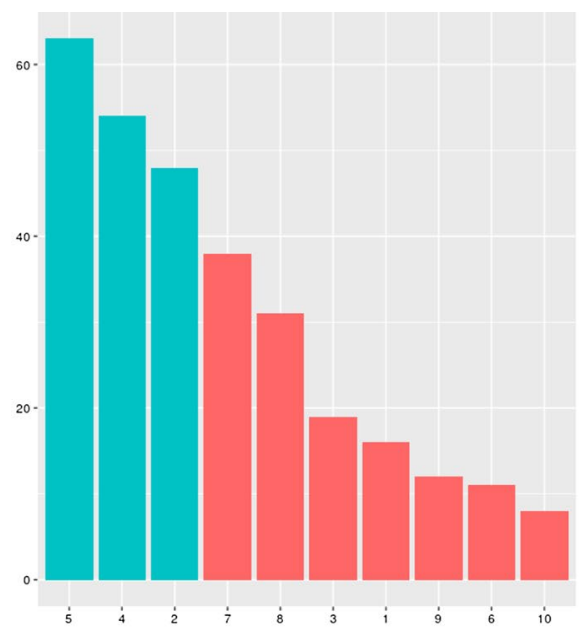

b Percent of selection of pathways for sgPLS on breast data, 100 bootstraps

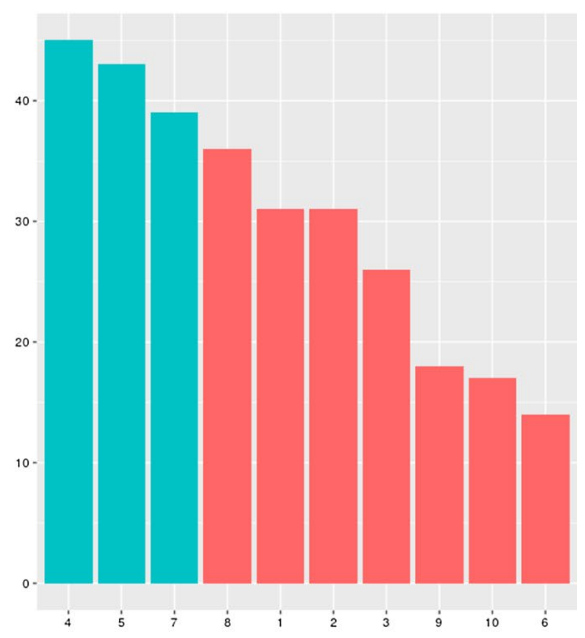

d Percent of selection of pathways for joint-sgPLS on all data, 100 bootstraps

Fig. 6 Percent of selection of pathways for sgPLS and joints sgPLS on 100 bootstraps. a sgPLS on thyroid data. $\mathbf{b}$ sgPLS on breast data. $\mathbf{c}$ sgPLS on both data. $\mathbf{d}$ joint-sgPLS. Pathways selected on original data (preselected ones) are in blue while other pathways (non-preselected ones) are in red. The pathways are noted: (1) Cell cycle (2) Circadian rhythm (3) Folate metabolism (4) Other glycan degradation (5) Obesity and obesity-related phenotypes (6) DNA repair (7) Metabolism of xenobiotics (9) Precocious or delayed puberty (10) Inflammatory response

Table 4 Computational performances in seconds of ASSET, metaSKAT, sgPLS and jointsgPLS for case $1(n=200)$ and case $2(n=400)$

\begin{tabular}{lll}
\hline $\mathbf{n}$ & $\mathbf{2 0 0}$ & $\mathbf{4 0 0}$ \\
\hline ASSET & 1.21 & 1.69 \\
metaSKAT & 0.22 & 0.59 \\
sgPLS & 18.29 & 37.03 \\
joint-sgPLS & 31.27 & 67.80 \\
\hline
\end{tabular}




\section{Computational performances}

Computation performances are presented on simulation cases 1 and 2 which represent data having 500 predictors and 1 output (Table 4). The number of observations is respectively $n=200$ and $n=400$. The methods sgPLS and joint-PLS penalization hyperparameters are estimated with the grid used for the simulation. Mean running times over 50 replications are given.

We can see overall that has the smallest running time. The methods sgPLS and jointsgPLS have the most expensive computational. This is due to the estimation of the penalization parameters as hyperparameters. However, this calculus consist in successive applications of the same method. It can then be paralellized.

\section{Discussion}

In this article, the properties of the joint-sgPLS are presented and are compared to the classical sgPLS, the ASSET method and metaSKAT. The methods ASSET, metaSKAT and joint-sgPLS are suited for meta-analyses whereas sgPLS is not. ASSET only gives variable-level results whereas metaSKAT and joint-sgPLS can assess group-level results. However, joint-sgPLS is the only method proposing to link in a same model variable results and group results. The method have then more interpretability while have competitive or superior performances over simulations compared to benchmark methods. Hence, joint-sgPLS seems perfectly suited for meta-analysis where effects in opposite directions can exist which invite us to pursue further investigation with it in complex studies for genetic epidemiology such as pleiotropy.

\section{Conclusion}

We do believe that further investigation can be done on the same subject. In this article, sgPLS and joint-sgPLS have been applied with one component, but several components could be considered. This could lead to the selection of variables that are orthogonal to the selection of the first component but that have still a large participation to the covariance matrix.

We acknowledge that on the application the stability of the method is an important point due to the fact that the cross-validation procedure is not satisfying for choosing the parameters of penalization. One improvement could consist in exploiting different the criteria of the procedure (the error prediction) with, for instance, stability measures [53]. Another improvement could consist in adaptating the adaptative Lasso [54] for our method which could bypass the stability questions.

Presented method uses a group architecture, but adding group-sub-group architecture is an interesting path of investigation for taking into account gene- and pathway-level information at the same time. The methods sgsPLS ( [36]) already offers a sparse partial least squares framework with group and subgroup architecture which is an extension of the sgPLS. A similar work could lead to a promising joint-sgsPLS.

In order to advance on the application, this study should be replicated on a larger data base. Particularly, thyroid cancer has been less studied than breast cancer, and data for thyroid are still scarce in this application. Other cases of pleiotropy could be investigated, for instance for the case where the phenotype is multivariate for each subject. The 
joint-sgPLS is suitable for any kind of phenotype, continuous or qualitative. $\mathrm{R}$ code is available from the author to reproduce the results and is available on github (https:// github.com/camilobroc/BMC_joint_sgPLS).

\begin{abstract}
Abbreviations
GWAS: Genome-wide association study; SNP: Single nucleotide polymorphism; MAF: Minor allele frequency; PLS: Partial least squares; sPLS: sparse partial least squares; sgPLS: sparse group partial least squares; sgsPLS: sparse group sparse partial least squares; SKAT: SNP Kernel Association Test; TP: True positive; FP: False positive; FN: False negative; TN: True negative.
\end{abstract}

\title{
Acknowledgements
}

The authors acknowledge Pascal Guénel for providing the breast and thyroid cancer data. The authors acknowledge also the calculus center MCIA (Mésocentre de Calcul Intensif Aquitain) for providing its facilities. The "Ligue contre le Cancer" is acknowledged as well for its support for Cross Cancer Genomic Investigation of Pleiotropy project.

\section{Authors' contributions}

$\mathrm{CB}$ and $\mathrm{BL}$ designed the novel method. $\mathrm{CB}$ implemented the analysis. $T T$ perfomed interpretation on real data. $\mathrm{CB}, \mathrm{TT}$ and BL wrote the manuscript. All authors read and approved the final manuscript.

\section{Funding}

This study was supported by the"Ligue contre le Cancer" for its Cross Cancer Genomic Investigation of Pleiotropy project.

\section{Availability of data and materials}

Code generating simulated data which has been analysed in this article is available at https://github.com/camilobroc/ BMC_joint_sgPLS. It is designed for R software (version 3.6.3 and higher). The application dataset "Beluhca" that has been used during the current study is available from the authors on reasonable request.

Ethics approval and consent to participate

Written informed consent for the present study was obtained from all participants. Study protocols were approved by the French ethic committees (CNIL, CCPPRB) (reference numbers 05-3144 for CATHY study and 04-53 for CECILE study).

\section{Consent for publication}

Not applicable.

\section{Competing interests}

The authors declare that they have no competing interests.

\section{Author details}

'LIST, CEA, Laboratory for Data Sciences and Decision (Digiteo), Gif-sur-Yvette, France. ${ }^{2}$ CNRS, Laboratoire de Mathématiques et de leurs Applications de PAU E2S UPPA, Pau, France. ${ }^{3}$ UVSQ, Inserm, CESP, Université Paris-Saclay, 94807 Villejuif, France. ${ }^{4}$ Institut Gustave Roussy, 94805 Villejuif, France. ${ }^{5}$ Department of Mathematics and Statistics, Macquarie University, Sydney, Australia.

\section{Appendix 1: Tables of genes and pathways}

\section{See Tables 5, 6 .}


Table 5 First pathways and their corresponding genes.

\begin{tabular}{|c|c|}
\hline Gene & Count \\
\hline \multicolumn{2}{|l|}{ (1) } \\
\hline ENPP1 & 27 \\
\hline NMNAT3 & 25 \\
\hline $\mathrm{AOX} 1$ & 24 \\
\hline NMNAT2 & 21 \\
\hline ENPP3 & 15 \\
\hline BST1 & 14 \\
\hline CD38 & 11 \\
\hline NT5M & 10 \\
\hline NT5C1A & 9 \\
\hline NT5C2 & 8 \\
\hline PNP & 8 \\
\hline NAMPT & 7 \\
\hline NNMT & 7 \\
\hline NT5C3 & 7 \\
\hline NADSYN1 & 6 \\
\hline NNT & 6 \\
\hline NT5E & 5 \\
\hline NMNAT1 & 4 \\
\hline NUDT12 & 4 \\
\hline QPRT & 4 \\
\hline NT5C1B & 3 \\
\hline NADK & 2 \\
\hline NT5C & 2 \\
\hline \multicolumn{2}{|l|}{ (2) } \\
\hline RORA & 282 \\
\hline NPAS2 & 60 \\
\hline RORB & 34 \\
\hline ARNTL & 23 \\
\hline CUL1 & 22 \\
\hline BTRC & 13 \\
\hline RORC & 13 \\
\hline PER3 & 12 \\
\hline CLOCK & 11 \\
\hline PER2 & 11 \\
\hline CRY2 & 9 \\
\hline CSNK1E & 9 \\
\hline BHLHE40 & 8 \\
\hline FBXW11 & 8 \\
\hline CRY1 & 7 \\
\hline FBXL3 & 7 \\
\hline NR1D1 & 7 \\
\hline TIMELESS & 7 \\
\hline PER1 & 5 \\
\hline SKP1 & 4 \\
\hline BHLHE41 & 3 \\
\hline CSNK1D & 3 \\
\hline RBX1 & 2 \\
\hline
\end{tabular}


Table 5 (continued)

\begin{tabular}{|c|c|}
\hline Gene & Count \\
\hline \multicolumn{2}{|l|}{ (3) } \\
\hline MTHFS & 19 \\
\hline MTHFD1 & 11 \\
\hline MTHFR & 11 \\
\hline MTHFD2L & 9 \\
\hline MTHFD2 & 1 \\
\hline \multicolumn{2}{|l|}{ (4) } \\
\hline GLB1 & 22 \\
\hline MAN2B2 & 18 \\
\hline ENGASE & 13 \\
\hline HEXB & 12 \\
\hline MANBA & 9 \\
\hline AGA & 8 \\
\hline FUCA2 & 8 \\
\hline NEU2 & 5 \\
\hline FUCA1 & 3 \\
\hline HEXA & 3 \\
\hline NEU3 & 3 \\
\hline GBA & 2 \\
\hline MAN2B1 & 2 \\
\hline NEU1 & 2 \\
\hline NEU4 & 1 \\
\hline \multicolumn{2}{|l|}{ (5) } \\
\hline LRRN6C & 197 \\
\hline FTO & 122 \\
\hline NEGR1 & 93 \\
\hline SCARB1 & 33 \\
\hline $\mathrm{ABCC} 8$ & 31 \\
\hline SEC16B & 25 \\
\hline LEPR & 23 \\
\hline MAP2K5 & 23 \\
\hline $\mathrm{NR3C1}$ & 18 \\
\hline FAIM2 & 17 \\
\hline DRD2 & 16 \\
\hline PPARG & 16 \\
\hline SIM1 & 14 \\
\hline FANCL & 12 \\
\hline GHRL & 12 \\
\hline ADIPOQ & 11 \\
\hline CRHR2 & 11 \\
\hline GNB3 & 10 \\
\hline INSR & 10 \\
\hline GPRC5B & 9 \\
\hline MC3R & 9 \\
\hline PCSK1 & 9 \\
\hline TFAP2B & 9 \\
\hline TNF & 9 \\
\hline UCP1 & 9 \\
\hline
\end{tabular}


Table 5 (continued)

\begin{tabular}{|c|c|}
\hline Gene & Count \\
\hline \multicolumn{2}{|l|}{ (5) } \\
\hline CRHR1 & 8 \\
\hline ETV5 & 8 \\
\hline IL1RN & 8 \\
\hline LDLR & 8 \\
\hline HTR2C & 7 \\
\hline MCHR1 & 7 \\
\hline TMEM18 & 7 \\
\hline BDNF & 6 \\
\hline KCTD15 & 6 \\
\hline UCP2 & 6 \\
\hline ACE & 5 \\
\hline ADRB2 & 4 \\
\hline IL6 & 4 \\
\hline LEP & 4 \\
\hline MC4R & 4 \\
\hline PLIN & 4 \\
\hline PTPN11 & 4 \\
\hline UCP3 & 4 \\
\hline GNPDA2 & 3 \\
\hline NROB2 & 3 \\
\hline RETN & 3 \\
\hline CCL5 & 2 \\
\hline LEPROTL1 & 2 \\
\hline $\mathrm{SH} 2 \mathrm{~B} 1$ & 1 \\
\hline \multicolumn{2}{|l|}{ (6) } \\
\hline RPA3 & 25 \\
\hline NEIL3 & 24 \\
\hline XRCC5 & 21 \\
\hline EXO1 & 19 \\
\hline $\mathrm{MSH} 3$ & 19 \\
\hline NEIL2 & 17 \\
\hline RPA1 & 17 \\
\hline BRCA2 & 16 \\
\hline RAD23B & 16 \\
\hline RFC3 & 14 \\
\hline PARP4 & 13 \\
\hline POLD3 & 12 \\
\hline RFC5 & 12 \\
\hline CHEK1 & 11 \\
\hline CHEK2 & 11 \\
\hline MSH6 & 11 \\
\hline TERT & 10 \\
\hline CASP7 & 9 \\
\hline MNAT1 & 9 \\
\hline PARP1 & 9 \\
\hline RFC1 & 9 \\
\hline XPC & 9 \\
\hline CASP3 & 8 \\
\hline
\end{tabular}


Table 5 (continued)

\begin{tabular}{|c|c|}
\hline Gene & Count \\
\hline ERCC5 & 8 \\
\hline ERCC6 & 8 \\
\hline \multicolumn{2}{|l|}{ (6) } \\
\hline TDG & 8 \\
\hline XRCC1 & 8 \\
\hline DDB2 & 7 \\
\hline ERCC8 & 7 \\
\hline $\mathrm{MSH} 2$ & 7 \\
\hline PMS2 & 7 \\
\hline POLD1 & 7 \\
\hline POLE & 7 \\
\hline RFC4 & 7 \\
\hline XRCC2 & 7 \\
\hline XRCC3 & 7 \\
\hline CDK2 & 6 \\
\hline GTF2H1 & 6 \\
\hline LIG1 & 6 \\
\hline MUTYH & 6 \\
\hline PARP2 & 6 \\
\hline POLD2 & 6 \\
\hline POLE2 & 6 \\
\hline TP53 & 6 \\
\hline XPA & 6 \\
\hline BRCA1 & 5 \\
\hline CDK7 & 5 \\
\hline CHRNA4 & 5 \\
\hline CUL4B & 5 \\
\hline ERCC3 & 5 \\
\hline
\end{tabular}

The number of SNPs for each gene is presented: The pathways are (1) Nicotinate and nicotinamide metabolism (2) Circadian rhythm (3) Folate metabolism (4) Other glycan degradation (5) Obesity and obesity-related phenotypes (6) DNA repair 
Table 6 Last pathways and their corresponding genes.

\begin{tabular}{|c|c|}
\hline Gene & Count \\
\hline \multicolumn{2}{|l|}{ (6) } \\
\hline GTF2H5 & 5 \\
\hline LIG3 & 5 \\
\hline MAPK8 & 5 \\
\hline NTHL1 & 5 \\
\hline OGG1 & 5 \\
\hline RAD50 & 5 \\
\hline RPA4 & 5 \\
\hline APEX1 & 4 \\
\hline CDKN1A & 4 \\
\hline ERCC2 & 4 \\
\hline FEN1 & 4 \\
\hline GTF2H4 & 4 \\
\hline MBD4 & 4 \\
\hline POLE3 & 4 \\
\hline RAD51 & 4 \\
\hline RFC2 & 4 \\
\hline UNG & 4 \\
\hline CDKN2D & 3 \\
\hline CETN2 & 3 \\
\hline CUL4A & 3 \\
\hline ERCC1 & 3 \\
\hline MLH1 & 3 \\
\hline MPG & 3 \\
\hline POLB & 3 \\
\hline POLE4 & 3 \\
\hline \multicolumn{2}{|l|}{ (6) } \\
\hline POLL & 3 \\
\hline RPA2 & 3 \\
\hline SSBP1 & 3 \\
\hline $\mathrm{CCNH}$ & 2 \\
\hline ERCC4 & 2 \\
\hline GTF2H3 & 2 \\
\hline HMGB1 & 2 \\
\hline \multicolumn{2}{|l|}{ (7) } \\
\hline UGT1A8 & 58 \\
\hline UGT2A1 & 29 \\
\hline MGST2 & 22 \\
\hline CYP2C8 & 17 \\
\hline AKR1C2 & 16 \\
\hline CYP2C9 & 15 \\
\hline CYP2B6 & 14 \\
\hline $\mathrm{EPHX} 1$ & 14 \\
\hline MGST3 & 14 \\
\hline AKR1C4 & 13 \\
\hline COMT & 12 \\
\hline CYP2C19 & 12 \\
\hline CYP2S1 & 12 \\
\hline CYP2C18 & 11 \\
\hline
\end{tabular}


Table 6 (continued)

\begin{tabular}{|c|c|}
\hline Gene & Count \\
\hline $\mathrm{ADH} 1 \mathrm{~B}$ & 10 \\
\hline $\mathrm{ADH7}$ & 10 \\
\hline GSTA4 & 10 \\
\hline GSTZ1 & 10 \\
\hline MGST1 & 10 \\
\hline NAT2 & 10 \\
\hline $\mathrm{ADH} 1 \mathrm{C}$ & 9 \\
\hline AHR & 9 \\
\hline NAT1 & 9 \\
\hline ADH6 & 8 \\
\hline AKR1C3 & 8 \\
\hline \multicolumn{2}{|l|}{ (7) } \\
\hline CYP1B1 & 8 \\
\hline UGT2B4 & 8 \\
\hline $\mathrm{ADH} 4$ & 7 \\
\hline ADH5 & 7 \\
\hline AKR1C1 & 7 \\
\hline ALDH3B1 & 7 \\
\hline CYP2E1 & 7 \\
\hline NQO1 & 7 \\
\hline ALDH1A3 & 6 \\
\hline CYP2F1 & 6 \\
\hline $\mathrm{DHDH}$ & 6 \\
\hline SOD2 & 6 \\
\hline GSTA2 & 5 \\
\hline GSTM3 & 5 \\
\hline GSTO2 & 5 \\
\hline GSTP1 & 5 \\
\hline CYP1A1 & 4 \\
\hline CYP1A2 & 4 \\
\hline CYP3A43 & 4 \\
\hline GSTA3 & 4 \\
\hline GSTM4 & 4 \\
\hline UGT2B7 & 4 \\
\hline AKR1A1 & 3 \\
\hline ALDH3B2 & 3 \\
\hline CYP2A6 & 3 \\
\hline \multicolumn{2}{|l|}{ (7) } \\
\hline CYP3A4 & 3 \\
\hline CYP3A7 & 3 \\
\hline GSTM2 & 3 \\
\hline GSTM5 & 3 \\
\hline UGT2A3 & 3 \\
\hline UGT2B11 & 3 \\
\hline $\mathrm{ADH} 1 \mathrm{~A}$ & 2 \\
\hline CYP2D6 & 2 \\
\hline \multicolumn{2}{|l|}{ (8) } \\
\hline TGFBR3 & 77 \\
\hline EBF2 & 60 \\
\hline
\end{tabular}


Table 6 (continued)

\begin{tabular}{|c|c|}
\hline Gene & Count \\
\hline BCAT1 & 51 \\
\hline VDR & 34 \\
\hline KAL1 & 26 \\
\hline TM7SF3 & 19 \\
\hline CASC1 & 17 \\
\hline FGFR1OP2 & 12 \\
\hline FGFR1 & 11 \\
\hline KRAS2 & 10 \\
\hline CCR3 & 8 \\
\hline KISS1 & 8 \\
\hline PROK2 & 7 \\
\hline LIF & 5 \\
\hline PROKR2 & 5 \\
\hline PTH1R & 4 \\
\hline NKX2-1 & 2 \\
\hline \multicolumn{2}{|l|}{ (9) } \\
\hline TGFB2 & 22 \\
\hline IL18RAP & 13 \\
\hline CYP4F11 & 12 \\
\hline $\mathrm{EPHX} 2$ & 12 \\
\hline IL7 & 11 \\
\hline |GFBP1 & 9 \\
\hline IGFBP3 & 9 \\
\hline IL17A & 9 \\
\hline IL10 & 8 \\
\hline IGFBP4 & 7 \\
\hline IL15 & 7 \\
\hline MMP25 & 7 \\
\hline IL16 & 6 \\
\hline IL12A & 5 \\
\hline IL13 & 5 \\
\hline IL18 & 5 \\
\hline IL19 & 5 \\
\hline IL2 & 5 \\
\hline IL9 & 5 \\
\hline PLA2G4B & 5 \\
\hline IL3 & 4 \\
\hline TGFB1 & 4 \\
\hline IL1B & 3 \\
\hline IL4 & 3 \\
\hline IL1A & 2 \\
\hline IL23A & 1 \\
\hline \multicolumn{2}{|l|}{ 10) } \\
\hline EGFR & 103 \\
\hline CCND3 & 29 \\
\hline MAPT & 19 \\
\hline MAP2K4 & 15 \\
\hline EGF & 11 \\
\hline MAP2K2 & 11 \\
\hline
\end{tabular}




\section{Table 6 (continued)}

\begin{tabular}{lc}
\hline Gene & Count \\
\hline GSK3B & 8 \\
PLA2G6 & 8 \\
PTEN & 8 \\
MAP2K1 & 6 \\
MYBL2 & 6 \\
AKT1 & 5 \\
MAP2K3 & 5 \\
FGFR3 & 3 \\
MAP2K7 & 3 \\
MAPK12 & 3 \\
TP5313 & 3 \\
CCNA2 & 2 \\
MAPK7 & 2
\end{tabular}

The number of SNPs for each gene is presented. The pathways are (6) DNA repair (7) Metabolism of xenobiotics (8)

Precocious or delayed puberty (9) Inflammatory response (10) Cell cycle

Received: 27 May 2020 Accepted: 14 January 2021

Published online: 24 February 2021

\section{References}

1. Paaby AB, Rockman MV. The many faces of pleiotropy. Trends Genet. 2013;29(2):66-73.

2. Gratten J, Visscher PM. Genetic pleiotropy in complex traits and diseases: implications for genomic medicine. Genome Med. 2016;8(1):78.

3. Solovieff N, Cotsapas C, Lee PH, Purcell SM, Smoller JW. Pleiotropy in complex traits: challenges and strategies. Nat. Rev. Genet. 2013;14(7):483.

4. Yang C, Li C, Wang Q, Chung D, Zhao H. Implications of pleiotropy: challenges and opportunities for mining big data in biomedicine. Front. Genet. 2015;6:229.

5. Gagnon-Bartsch JA, Speed TP. Using control genes to correct for unwanted variation in microarray data. Biostatistics. 2012;13(3):539-52.

6. Bhattacharjee S, Rajaraman P, Jacobs KB, Wheeler WA, Melin BS, Hartge P, Yeager M, Chung CC, Chanock SJ, Chatterjee $\mathrm{N}$, et al. A subset-based approach improves power and interpretation for the combined analysis of genetic association studies of heterogeneous traits. Am J Human Genet. 2012;90(5):821-35.

7. Lee S, Teslovich TM, Boehnke M, Lin X. General framework for meta-analysis of rare variants in sequencing association studies. Am J Human Genet. 2013;93(1):42-53.

8. Pasaniuc B, Price AL. Dissecting the genetics of complex traits using summary association statistics. Nat Rev Genet. 2017;18(2):117-27.

9. Watanabe K, Stringer S, Frei O, Mirkov MU, de Leeuw C, Polderman TJ, van der Sluis S, Andreassen OA, Neale BM, Posthuma D. A global overview of pleiotropy and genetic architecture in complex traits. Nat Genet. 2019;51(9):1339-48.

10. Jang S-K, Saunders G, Liu M, Jiang Y, Liu DJ, Vrieze S. 23andMe Research Team, et al.: Genetic correlation, pleiotropy, and causal associations between substance use and psychiatric disorder. Psychol Med. 2020;1-11

11. Neale BM, Rivas MA, Voight BF, Altshuler D, Devlin B, Orho-Melander M, Kathiresan S, Purcell SM, Roeder K, Daly MJ. Testing for an unusual distribution of rare variants. PLoS Genet. 2011;7(3):1001322.

12. Lee $S$, Abecasis GR, Boehnke M, Lin X. Rare-variant association analysis: study designs and statistical tests. Am J Human Genet. 2014;95(1):5-23.

13. Chen Z, Wang K. Gene-based sequential burden association test. Stat Med. 2019;38(13):2353-63.

14. Lê Cao K-A, Boitard S, Besse P. Sparse pls discriminant analysis: biologically relevant feature selection and graphical displays for multiclass problems. BMC Bioinform. 2011;12(1):253.

15. Cai TT, Ma Z, Wu Y, et al. Sparse pca: Optimal rates and adaptive estimation. Annals Stat. 2013;41(6):3074-110.

16. Shahbaba B, Tibshirani R, Shachaf CM, Plevritis SK. Bayesian gene set analysis for identifying significant biological pathways. J R Stat Soc Ser C (Applied Statistics). 2011;60(4):541-57.

17. Evangelou E, loannidis JP. Meta-analysis methods for genome-wide association studies and beyond. Nat Rev Genet. 2013;14(6):379-89.

18. Mukhopadhyay I, Feingold E, Weeks DE, Thalamuthu A. Association tests using kernel-based measures of multi-locus genotype similarity between individuals. Genetic Epidemiol. 2010;34(3):213-21. 
19. Schweiger R, Weissbrod O, Rahmani E, Müller-Nurasyid M, Kunze S, Gieger C, Waldenberger M, Rosset S, Halperin E. Rl-skat: an exact and efficient score test for heritability and set tests. Genetics. 2017;207(4):1275-83.

20. Geng P, Tong X, Lu Q. An integrative u method for joint analysis of multi-level omic data. BMC Genet. 2019;20(1):1-12.

21. Fan R, Wang Y, Boehnke M, Chen W, Li Y, Ren H, Lobach I, Xiong M. Gene level meta-analysis of quantitative traits by functional linear models. Genetics. 2015;200(4):1089-104.

22. Jiang Y, Chiu C-Y, Yan Q, Chen W, Gorin MB, Conley YP, Lakhal-Chaieb ML, Cook RJ, Amos CI, Wilson AF. et al. Genebased association testing of dichotomous traits with generalized linear mixed models using extended pedigrees: applications to age-related macular degeneration. J Am Stat Assoc (just-accepted). 2020;1-35

23. Kim E, Dede M, Lenoir WF, Wang G, Srinivasan S, Colic M, Hart T. A network of human functional gene interactions from knockout fitness screens in cancer cells. Life Sci Alliance. 2019;2(2).

24. Yao C, Chen BH, Joehanes R, Otlu B, Zhang X, Liu C, Huan T, Tastan O, Cupples LA, Meigs JB, et al. Integromic analysis of genetic variation and gene expression identifies networks for cardiovascular disease phenotypes. Circulation. 2015;131(6):536-49.

25. Consortium G, et al. The genotype-tissue expression (gtex) pilot analysis: Multitissue gene regulation in humans. Science. 2015;348(6235):648-60.

26. Manzoni C, Kia DA, Vandrovcova J, Hardy J, Wood NW, Lewis PA, Ferrari R. Genome, transcriptome and proteome: the rise of omics data and their integration in biomedical sciences. Briefings Bioinform. 2018;19(2):286-302.

27. Vizcaíno JA, Csordas A, Del-Toro N, Dianes JA, Griss J, Lavidas I, Mayer G, Perez-Riverol Y, Reisinger F, Ternent T, et al. 2016 update of the pride database and its related tools. Nucleic Acids Res. 2015:44(D1):447-56.

28. Gamazon ER, Wheeler HE, Shah KP, Mozaffari SV, Aquino-Michaels K, Carroll RJ, Eyler AE, Denny JC, Nicolae DL, Cox NJ, et al. A gene-based association method for mapping traits using reference transcriptome data. Nat Genet. 2015;47(9):1091.

29. Wold H. Path models with latent variables: The nipals approach. 1975;307-357.

30. Boulesteix A-L, Strimmer K. Partial least squares: a versatile tool for the analysis of high-dimensional genomic data. Briefings Bioinform. 2006;8(1):32-44.

31. Nørgaard L, Saudland A, Wagner J, Nielsen JP, Munck L, Engelsen SB. Interval partial least-squares regression (i pls): a comparative chemometric study with an example from near-infrared spectroscopy. Appl Spectrosc. 2000;54(3):413-9.

32. Giessing C, Fink GR, Rösler F, Thiel CM. fmri data predict individual differences of behavioral effects of nicotine: a partial least square analysis. J Cogniti Neurosci. 2007;19(4):658-70.

33. Pearson K. Liii. on lines and planes of closest fit to systems of points in space. Lond Edinburgh Dublin Philos Mag J Sci. 1901:2(11):559-72.

34. Lê Cao K-A, Rossouw D, Robert-Granié C, Besse P. A sparse pls for variable selection when integrating omics data. Stat Appl Genet Mol Biol. 2008;7(1).

35. Liquet B, de Micheaux PL, Hejblum BP, Thiébaut R. Group and sparse group partial least square approaches applied in genomics context. Bioinformatics. 2015;32(1):35-42.

36. Sutton $M$, Thiébaut $R$, Liquet B. Sparse partial least squares with group and subgroup structure. Stat Med. 2018;37(23):3338-56.

37. Obozinski G, Taskar B, Jordan MI. Joint covariate selection and joint subspace selection for multiple classification problems. Stat Comput. 2010;20(2):231-52.

38. Broc C, Calvo B, Liquet B. Penalized partial least square applied to structured data. Arabian J Math. 2019;1-16.

39. de Lafaye Micheaux, P, Liquet B, Sutton M, et al. Pls for big data: a unified parallel algorithm for regularised group pls. Stat Surv. 2019;13:119-49.

40. Eslami A, Qannari EM, Kohler A, Bougeard S. Algorithms for multi-group pls. J Chemometr. 2014;28(3):192-201.

41. Wang T, Ho G, Ye K, Strickler H, Elston RC. A partial least-square approach for modeling gene-gene and geneenvironment interactions when multiple markers are genotyped. Genetic Epidemiol. 2009;33(1):6-15.

42. Shen $\mathrm{H}$, Huang JZ. Sparse principal component analysis via regularized low rank matrix approximation. J Multivar Anal. 2008;99(6):1015-34.

43. Vinzi VE, Trinchera L, Amato S. Pls path modeling from foundations to recent developments and open issues for model assessment and improvement. 2010;47-82.

44. Geladi P, Kowalski BR. Partial least-squares regression: a tutorial. Anal Chim Acta. 1986;185:1-17.

45. Colombani C, Croiseau P, Fritz S, Guillaume F, Legarra A, Ducrocq V, Robert-Granié C. A comparison of partial least squares (pls) and sparse pls regressions in genomic selection in french dairy cattle. J Dairy Sci. 2012;95(4):2120-31.

46. d'Aspremont A, Ghaoui LE, Jordan MI, Lanckriet GR. A direct formulation for sparse pca using semidefinite programming. In: Advances in Neural Information Processing Systems. 2005;pp. 41-48

47. Jacob L, Obozinski G, Vert J-P. Group lasso with overlap and graph lasso. In: Proceedings of the 26th Annual International Conference on Machine Learning. 2009;pp. 433-440. ACM

48. Nielsen SM, White MG, Hong S, Aschebrook-Kilfoy B, Kaplan EL, Angelos P, Kulkarni SA, Olopade Ol, Grogan RH. The breast-thyroid cancer link: a systematic review and meta-analysis. Cancer Epidemiol Prevent Biomarkers. 2016:25(2):231-8

49. Mavaddat N, Michailidou K, Dennis J, Lush M, Fachal L, Lee A, Tyrer JP, Chen T-H, Wang Q, Bolla MK, et al. Polygenic risk scores for prediction of breast cancer and breast cancer subtypes. Am J Human Genet. 2019;104(1):21-34.

50. Czene K, Lichtenstein P, Hemminki K. Environmental and heritable causes of cancer among 9.6 million individuals in the swedish family-cancer database. Int J Cancer. 2002;99(2):260-6.

51. Gudmundsson J, Thorleifsson G, Sigurdsson JK, Stefansdottir L, Jonasson JG, Gudjonsson SA, Gudbjartsson DF, Masson $\mathrm{G}$, Johannsdottir $\mathrm{H}$, Halldorsson $\mathrm{GH}$, et al. A genome-wide association study yields five novel thyroid cancer risk loci. Nat Commun. 2017:8:14517. 
52. Stacey SN, Manolescu A, Sulem P, Rafnar T, Gudmundsson J, Gudjonsson SA, Masson G, Jakobsdottir M, Thorlacius S, Helgason A, et al. Common variants on chromosomes 2 q35 and $16 \mathrm{q} 12$ confer susceptibility to estrogen receptorpositive breast cancer. Nat Genet. 2007;39(7):865.

53. Nogueira S, Sechidis K, Brown G. On the stability of feature selection algorithms. J Mach Learn Res. 18, 174-1

54. Zou H. The adaptive lasso and its oracle properties. J Am Stat Assoc. 2006;101(476):1418-29.

\section{Publisher's Note}

Springer Nature remains neutral with regard to jurisdictional claims in published maps and institutional affiliations.

- fast, convenient online submission

- thorough peer review by experienced researchers in your field

- rapid publication on acceptance

- support for research data, including large and complex data types

- gold Open Access which fosters wider collaboration and increased citations

- maximum visibility for your research: over 100M website views per year

At BMC, research is always in progress.

Learn more biomedcentral.com/submissions 